\title{
Fine tuning the extracellular environment accelerates the derivation of kidney organoids from human pluripotent stem cells
}

\author{
Elena Garreta1,13, Patricia Prado 1,13, Carolina Tarantino', Roger Oria ${ }^{2,3}$, Lucia Fanlo4, Elisa Martí ${ }^{4}$, \\ Dobryna Zalvidea ${ }^{5}$, Xavier Trepat ${ }^{5,6,7,8}$, Pere Roca-Cusachs ${ }^{2,3}$, Aleix Gavaldà-Navarro ${ }^{9}$, Luca Cozzuto ${ }^{10}$, \\ Josep Maria Campistol ${ }^{11}$, Juan Carlos Izpisúa Belmonte ${ }^{12}$, Carmen Hurtado del Pozo ${ }^{1}$ and \\ Nuria Montserrat $\mathbb{C}^{1,6 \star}$
}

The generation of organoids is one of the biggest scientific advances in regenerative medicine. Here, by lengthening the time that human pluripotent stem cells (hPSCs) were exposed to a three-dimensional microenvironment, and by applying defined renal inductive signals, we generated kidney organoids that transcriptomically matched second-trimester human fetal kidneys. We validated these results using ex vivo and in vitro assays that model renal development. Furthermore, we developed a transplantation method that utilizes the chick chorioallantoic membrane. This approach created a soft in vivo microenvironment that promoted the growth and differentiation of implanted kidney organoids, as well as providing a vascular component. The stiffness of the in ovo chorioallantoic membrane microenvironment was recapitulated in vitro by fabricating compliant hydrogels. These biomaterials promoted the efficient generation of renal vesicles and nephron structures, demonstrating that a soft environment accelerates the differentiation of hPSC-derived kidney organoids.

K idney organoids have been produced from human pluripotent stem cells (hPSCs) by specific induction of the metanephric mesenchyme (MM) lineage (including nephron progenitor cells, NPCs $)^{1-7}$, or by the simultaneous induction of MM- and ureteric bud (UB)-like progenitors ${ }^{8,9}$, the two progenitor cell populations that give rise to the adult kidney during development. Recently, NPCs and UB progenitors were separately induced and then aggregated together into three-dimensional (3D) spheroids that generated kidney organoids with higher-order architecture ${ }^{10}$. For kidney organoids generated from human embryonic stem cells (hESCs), CRISPR/Cas9 technology can be used to recapitulate the molecular features of kidney diseases ${ }^{4}$. Human kidney organoids can also be used as unprecedented in vitro models to screen for nephrotoxicity ${ }^{3,4,9}$. Besides the importance of these findings, major concerns related to the lack of vascularization and insufficient maturation still require further investigation to advance the field of hPSC-derived kidney organoids (kidney organoids). Biophysical cues have been shown to regulate cell behaviour, including the stemness and differentiation of different stem cell populations. Recently, application of fluid flow enhanced hPSC-derived podocyte-like cell differentiation in monolayer culture ${ }^{11}$, and the modulation of adherent forces in kidney organoids, resulted in changes in the functional performance of proximal tubular epithelial-like cells within kidney organoids ${ }^{12}$.

Efficient generation of kidney organoids in 3D culture During mammalian kidney development, the posterior primitive streak (PPS) and anterior primitive streak (APS) give rise to the intermediate mesoderm (IM) and definitive endoderm, respectively. The posterior IM generates the MM, whereas the anterior IM forms the UB. The PPS can be generated from hPSCs using a combination of growth factors (including BMP4) ${ }^{1,7,8}$, or by exposing undifferentiated cells to varying doses and durations of the Wnt signalling agonist CHIR99021 (CHIR), a widely used inhibitor of glycogen synthase kinase $3(\mathrm{GSK} 3 \beta)^{1-5,8,9}$. Building upon these observations, we asked whether PPS cells could be generated by exposing hPSCs to a high dose of CHIR $(8 \mu \mathrm{M})$ in two-dimensional monolayer culture over three consecutive days (Supplementary Fig. 1a; Methods). This treatment regimen was sufficient to induce PPS-committed cells that were positive for the PPS marker BRACHYURY (referred to as T), at $82.2 \pm 2.6 \%$ efficiency (Supplementary Fig. 1b,c). PPScommitted cells upregulated the expression of PPS genes in comparison with APS genes (Supplementary Fig. 1d). Subsequent exposure of PPS-committed cells to a combination of FGF9 and activin A

\footnotetext{
'Pluripotency for Organ Regeneration, Institute for Bioengineering of Catalonia (IBEC), The Barcelona Institute of Technology (BIST), Barcelona, Spain. ${ }^{2}$ Cellular and Respiratory Biomechanics, Institute for Bioengineering of Catalonia (IBEC), The Barcelona Institute of Technology (BIST), Barcelona, Spain. ${ }^{3}$ University of Barcelona, Barcelona, Spain. ${ }^{4}$ Instituto de Biología Molecular de Barcelona (IBMB-CSIC), Parc Científic de Barcelona, Barcelona, Spain. ${ }^{5}$ Institute for Bioengineering of Catalonia (IBEC), The Barcelona Institute of Technology (BIST), Barcelona, Spain. ${ }^{6} \mathrm{Centro}$ de Investigación Biomédica en Red en Bioingeniería, Biomateriales y Nanomedicina, Barcelona, Spain. ${ }^{7}$ Unitat de Biofísica i Bioenginyeria, Universitat de Barcelona, Barcelona, Spain. ${ }^{8}$ Institució Catalana de Recerca i Estudis Avançats (ICREA), Barcelona, Spain. 'Departament de Bioquímica i Biomedicina Molecular, Institut de Biomedicina (IBUB), Universitat de Barcelona and CIBER Fisiopatología de la Obesidad y Nutrición, Barcelona, Spain. ${ }^{10}$ Centre for Genomic Regulation (CRG), The Barcelona Institute of Science and Technology, Barcelona, Spain. " Hospital Clinic, University of Barcelona, IDIBAPS, Barcelona, Spain. ${ }^{12}$ Gene Expression Laboratory, Salk Institute for Biological Studies, La Jolla, CA, USA. ${ }^{13}$ These authors contributed equally: Elena Garreta and Patricia Prado. *e-mail: nmontserrat@ibecbarcelona.eu
} 
(20:1) for an additional day resulted in acquisition of the early IM marker PAX2 at $85.0 \pm 1.4 \%$ efficiency (Supplementary Fig. 2a-c; Methods). Accordingly, messenger RNA levels for the posterior IM markers OSR1 and HOXD11 and the anterior IM marker GATA3 were also upregulated at this stage (Supplementary Fig. 2d).

We next reasoned that increasing the time that IM-committed cells are exposed to 3D culture, which increase cell-to-cell and cellto-extracellular matrix interactions, would generate kidney organoids at higher efficiencies than previously reported ${ }^{3,8,9}$. Therefore, $3 \mathrm{D}$ spheroids were generated by the self-aggregation of IM cells and maintained under 3D organotypic culture until day 16 (Fig. 1a; see Methods). Treatment of IM-committed 3D spheroids (day 0) with CHIR $(3 \mu \mathrm{M})$ for $3 \mathrm{~d}$ (from day 0 to day 3 ), while maintaining FGF9 signalling (from day 0 to day 7 ), resulted in the formation of numerous renal vesicles (RVs) on day 8 (Supplementary Fig. 3a,b), which were analysed by immunofluorescence for RV-associated markers, including PAX2, WT1, LHX1, PAX8, HNF1 $\beta$, ECAD and BRN1 (Supplementary Fig. 3a,c,d). Remarkably, SIX2-positive cells were absent on day 8 , indicating the lack of MM progenitors at this stage (Supplementary Fig. 3d). In addition, downregulation of the epithelial-to-mesenchymal markers TWIST and SNAIL and upregulation of WNT4 and ECAD coincided with initiation of the mesenchymal-to-epithelial transition, which is essential for nephrogenesis (Supplementary Fig. 3e). RV-stage organoids were then differentiated in the absence of growth factors (from day 7 to day 16). This resulted in the derivation of kidney organoids with multiple nephron-like structures that were segmented into typical nephron components, including proximal tubules $\left(\mathrm{LTL}^{+} \mathrm{AQP1}^{+} / \mathrm{SLC} \mathrm{A} 1^{+}\right)$, loops of Henle $\left(\mathrm{ECAD}^{+} \mathrm{UMOD}^{+}\right)$, distal tubules $\left(\mathrm{UMOD}^{-} \mathrm{ECAD}^{+}\right)$, and glomeruli $\left(\mathrm{PODXL}^{+} / \mathrm{PODOCIN}^{+} / \mathrm{NEPHRIN}^{+} / \mathrm{NEPH}^{+} / \mathrm{WT}^{+}{ }^{+} \mathrm{PODXL}^{+}\right.$ $\mathrm{LTL}^{-} / \mathrm{PODOCIN}^{+} \mathrm{LTL}^{-}$) (Fig. 1b, Supplementary Fig. 4a-g). In addition, quantitative PCR (qPCR) and immunofluorescence analyses confirmed that isolated $\mathrm{LTL}^{-}$and $\mathrm{LTL}^{+}$cell fractions from day 16 organoids expressed markers of glomerular and proximal tubular identity, respectively (Supplementary Fig. 5a-f). Similarly, markers representative of the major steps of differentiation were analysed by qPCR (Supplementary Fig. 6). Our methodology was quite robust, as kidney organoids from two commercial hESClines and one human induced pluripotent stem cell (hiPSC) line were also generated (Supplementary Fig. 7a-d, Supplementary Fig. 8).

To gain insight into the sequence of transcription regulatory events necessary to promote renal differentiation from hPSCs, we performed RNA sequencing (RNA-Seq) analysis at major stages during the differentiation process. We compared our results with transcriptional data from human fetal organs/tissues from the first and second trimesters of gestation ${ }^{13,14}$ (Supplementary Fig. 9, Supplementary Table 1; Methods), demonstrating that our technique specifically generated renal lineages. Importantly, RNA-Seq analysis showed that day $8 \mathrm{RV}$-stage organoids transcriptionally matched human fetal kidneys at 16 weeks of gestation, whereas day 16 kidney organoids matched human fetal kidneys at 22 weeks of gestation (Fig. 1c, Supplementary Table 2; Methods). We validated these findings by analysing markers of nephron progenitors (SIX2, $S A L L 1, P A X 2)$, the proximal tubular segment (SLC3A1) and the glomerular compartment (NPHS1, PODXL, SYNPO, WT1) via qPCR (Fig. 1d). Additionally, immunofluorescence analysis showed that localization of late-stage nephron markers was comparable between kidney organoids and human fetal kidney samples (Supplementary Fig. 4a-g). Transmission electron microscopy (TEM) analysis was performed on day 16 kidney organoids (Fig. 1e-j). Ultrathin sections revealed the presence of primitive podocyte-like cells with deposition of a basement membrane (Fig. 1g) and developing primary and secondary cell processes (Fig. 1h). Epithelial tubular-like cells with brush borders and high mitochondrial content were also detected (Fig. 1i,j).
Kidney organoids recapitulate human kidney development

The formation of kidney organoids with segmented nephrons may depend on the existence of a transient population of NPCs responsible for the generation of nephron structures in vitro. We analysed by immunofluorescence the expression of OSR1, WT1, PAX2 and SIX2, confirming that cells exhibiting a NPC signature were present in day 5 spheroids (Supplementary Fig. 10a,b). The posterior origin of NPCs was also confirmed by the detection of HOXD11, OSR1, and WT1 mRNA by qPCR (Supplementary Fig. 10c). Interestingly, the anterior IM fate was also generated at this stage, as GATA3 mRNA was detected (Supplementary Fig. 10c). We next evaluated the ability of day 5 NPCs to form kidney chimaeric structures ex vivo, taking advantage of a faithful reaggregation assay with mouse embryonic kidney cells ${ }^{15,16}$. After $6 \mathrm{~d}$ of culture, differentiated NPCs, identified by the expression of human nuclear antigen $(\mathrm{HuNu})$, integrated into nascent nephron structures that expressed WT1 in the glomerular segment and PAX8 in the nascent nephron (Fig. 2a-f; Methods). Thus, day 5 NPCs exhibited the capacity to integrate into mouse nascent nephron structures, but not into the UB compartment, suggesting that the induction of UB derivatives from hPSCs may depend on additional exogenous signals. These results challenge previous findings ${ }^{9}$ and agreed with a recent study that identified optimal time windows and exogenous signals for selectively inducing NPC and UB lineages from mouse and human PSCs ${ }^{10}$.

Next, we investigated the capacity of human kidney organoids to faithfully recapitulate complex nephron patterning events that have been mainly studied in the mouse model ${ }^{17}$. Day $8 \mathrm{RV}$-stage organoids were exposed to inhibitors of tankynase (IWR1) and GSK3 $\beta$ (CHIR) to decrease or increase $\beta$-catenin signalling, respectively. CHIR treatment reduced the number of $\mathrm{WT}^{+}$glomeruluslike structures when compared with vehicle (control) and IWR1. In contrast, the percentage of $\mathrm{LTL}^{+}$proximal tubule-like structures was unchanged (Fig. 2g,h). qPCR analysis confirmed a decrease in the expression of $W T 1$ and PODXL (proximal segment), and the induction of WNT4 (a $\beta$-catenin target gene) in CHIR-treated organoids relative to control, whereas PAX2 (whole nephron) remained unchanged (Fig. 2i). We next determined the effect of disrupting Notch signalling by treating day $8 \mathrm{RV}$-stage organoids with the $\gamma$-secretase inhibitor DAPT. Inhibition of Notch resulted in a severe loss of proximal tubule-like structures $\left(\mathrm{LTL}^{+}\right)$, together with a reduction in $\mathrm{PODXL}^{+}$glomerulus-like structures when compared with control (Supplementary Fig. 11a,b). qPCR analysis confirmed the downregulation of proximal (WT1) and medial (SLC3A1) nephron segment markers (Supplementary Fig. 11c). These findings agree with the role of Notch signalling in specifying proximal and medial identity during nephron patterning in the mouse ${ }^{17}$, and expand previous knowledge about the effect of Notch signalling on kidney organoids ${ }^{3}$.

The kidney is a highly metabolic organ that generates ATP through oxidative phosphorylation. Into the light of this knowledge, we hypothesized that the energy metabolism profile of cells should be taken into account to promote the differentiation of hPSCs into renal subtypes. Therefore, we exposed day $8 \mathrm{RV}$-stage organoids to either cell culture medium that promotes glycolysis in stem cells ${ }^{18}$ (endothelial cell growth medium, EGM) or cell culture medium favouring oxidative phosphorylation (renal growth medium (REGM) with insulin) for $8 \mathrm{~d}$. Seahorse analysis revealed that REGM increased mitochondrial respiration in kidney organoids when compared with EGM (Fig. 2j-1), promoting an oxidative phosphorylation bioenergetic phenotype. Kidney organoids under REGM conditions enhanced tubule differentiation, as shown by the development of prominent proximal tubular structures $\left(\mathrm{LTL}^{+}\right)$, more than with EGM (Fig. $2 \mathrm{~m}, \mathrm{n}$ ), in agreement with previous findings ${ }^{19}$. 
a

\begin{tabular}{|c|c|c|c|c|c|c|}
\hline \multicolumn{3}{|c|}{ Monolayer culture } & \multicolumn{3}{|c|}{ Organotypic culture } & \\
\hline VTN/E8 & $8 \mu \mathrm{M} \mathrm{CHIR}$ & \begin{tabular}{|l} 
Act A \\
FGF9
\end{tabular} & $\begin{array}{c}3 \mu \mathrm{M} \text { CHIR } \\
\text { FGF9 }\end{array}$ & FGF9 & No growth factors & \\
\hline hPSC & PPS induction & IM & NPC induction & RV induction & Nephrogenesis & \\
\hline & & & & & & $\overrightarrow{D 16}$ \\
\hline
\end{tabular}

b

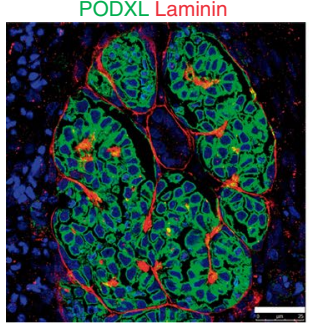

C

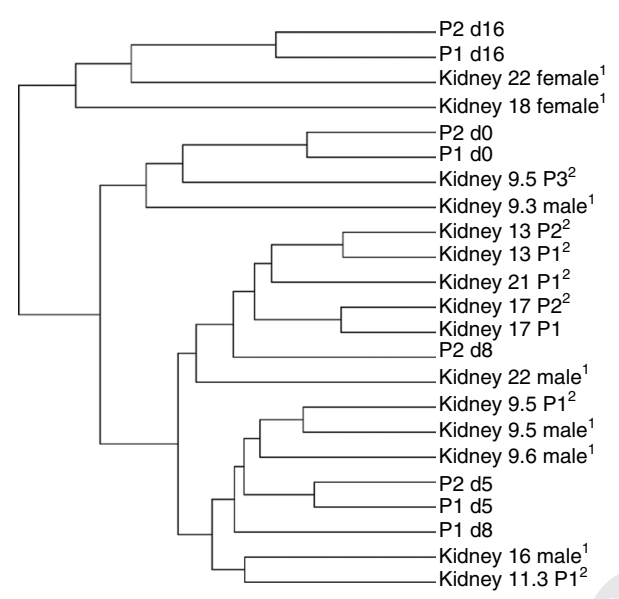

PODXL Podocin

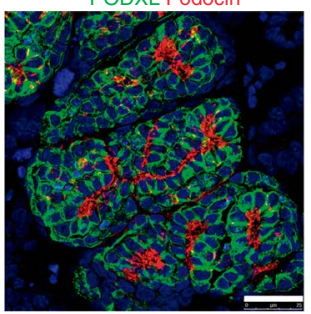

NEPHRIN Laminin

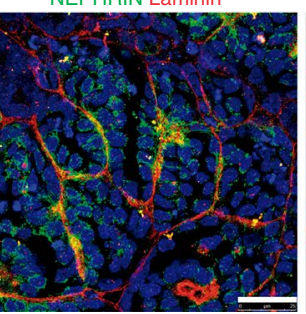

NEPH1 Laminin



d

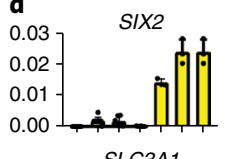

SLC3A1

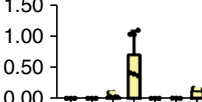

0.00 r SYNPO

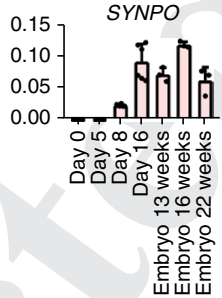

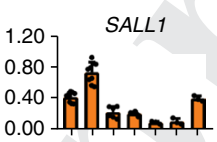

NPHS1

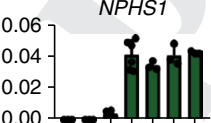

WT1

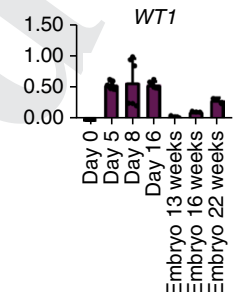

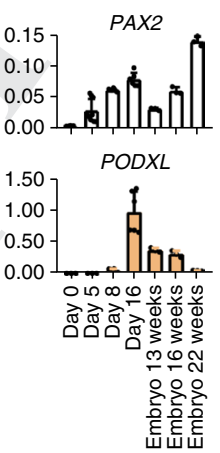

e


g
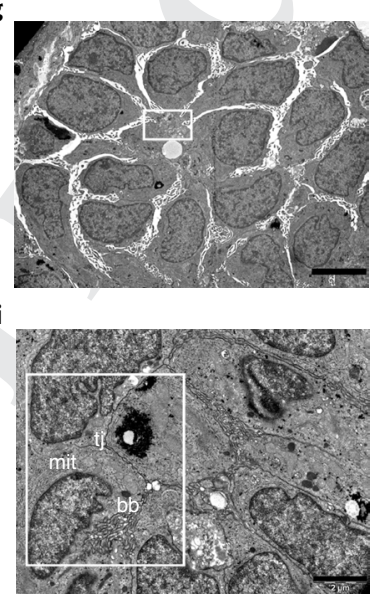

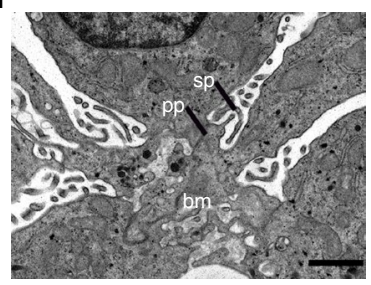

j

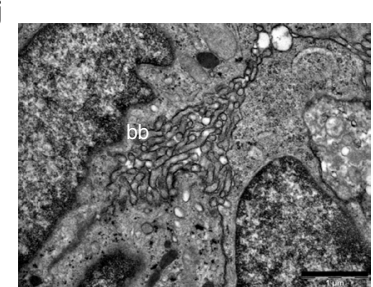

Fig. 1 | Efficient generation of kidney organoids in 3D culture. a, Schematic of the stepwise differentiation methodology for generating kidney organoids from hPSCs. b, Confocal microscopy images of glomerular structures in day 16 kidney organoids showing podocyte-like cells positive for PODXL, nephrin, $\mathrm{NEPH} 1$ and podocin, and the basement membrane protein laminin. Scale bars, $25 \mu \mathrm{m}$. c, Dendrogram representing the hierarchical clustering of day $0,5,8$ and 16 kidney organoids with human fetal kidneys from 9, 13, 17 and 18 weeks of gestation (first trimester) and 22 weeks of gestation (second trimester). Data from Chuva de Sousa Lopes (SRP055513) ${ }^{13}$ (1) and McMahon (SRP111183) ${ }^{14}$ (2) are included in the analysis. d, qPCR analysis during kidney organoid differentiation and 13-, 16-, and 22-week human fetal kidneys (genes are indicated). Data are mean \pm s.d. For SIX2, WT1, SALL1 and PAX2, day 0, day 5, $n=3$; day 8 , day $16, n=2$. For PODXL, SLC3A1, SYNPO and NPHS1, day 0 , day $5, n=1$; day 8 , day $16, n=2$. Each sample is a pool of six organoids. Three technical replicates are shown per sample. e,f, Semithin sections of day 16 kidney organoids showing glomerular (e) and tubular-like (f) structures. Scale bars, $100 \mu \mathrm{m}$. g-j, TEM of day 16 kidney organoids. $\mathbf{g}$, Immature podocytes. Scale bar, $5 \mu \mathrm{m}$. $\mathbf{h}$, A magnified view of the boxed region in $\mathbf{g}$ showing a detail of podocyte-related structures including the deposition of a basement membrane (bm), and primary (pp) and secondary cell processes (sp). Scale bar, $1 \mu \mathrm{m}$. i, Epithelial tubular-like cells with brush borders (bb), high mitochondrial (mit) content and tight junctions (tj). Scale bar, $2 \mu \mathrm{m}$. j, A magnified view of the boxed region in $\mathbf{i}$ showing a detail of brush borders. Scale bar, $1 \mu \mathrm{m}$. 
a

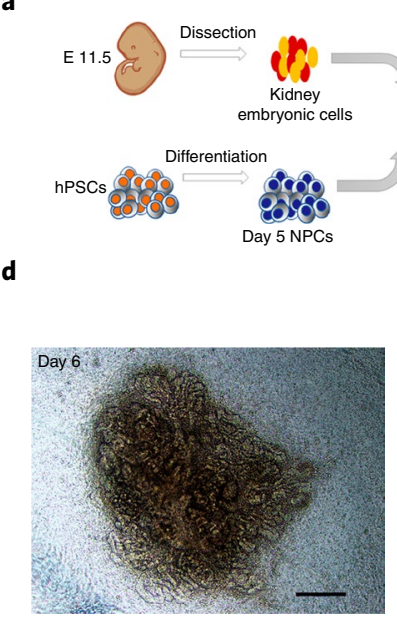

b

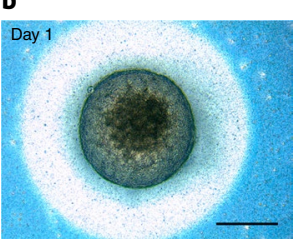

e

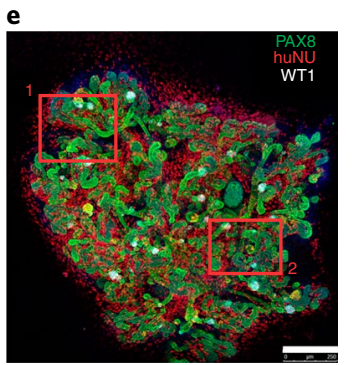

f 1 glomerular

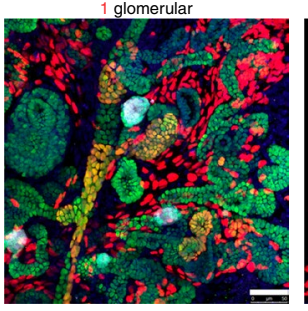

c

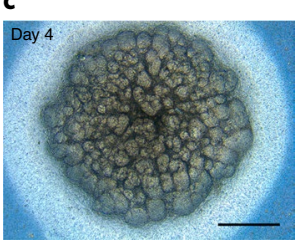

g

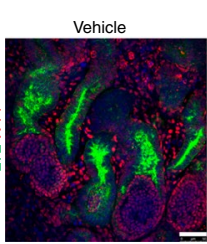

IWR1 5 uM
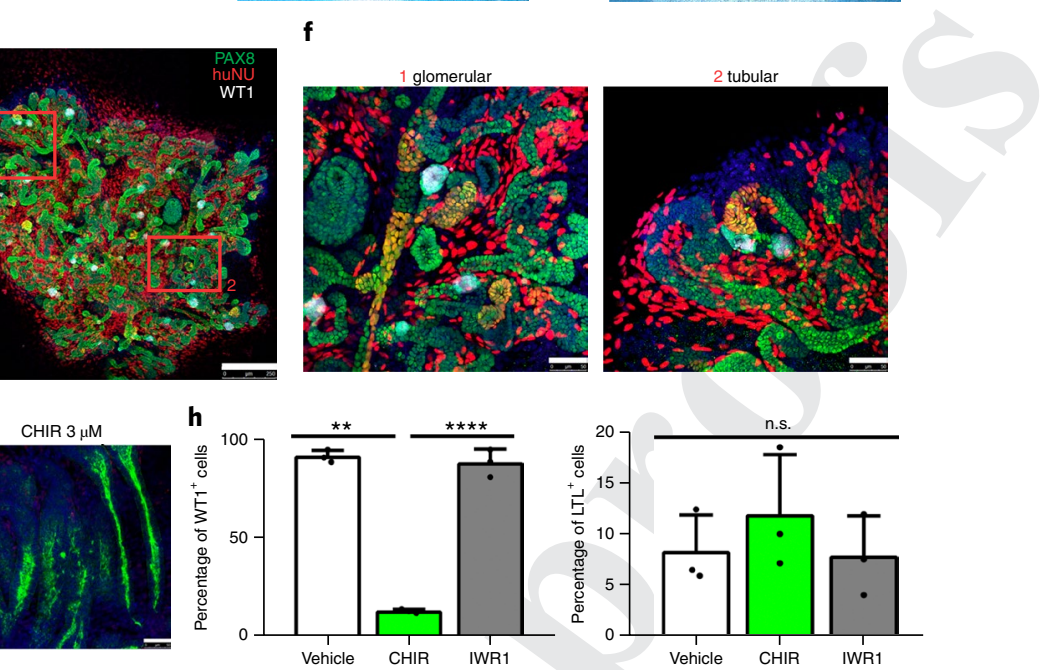

i
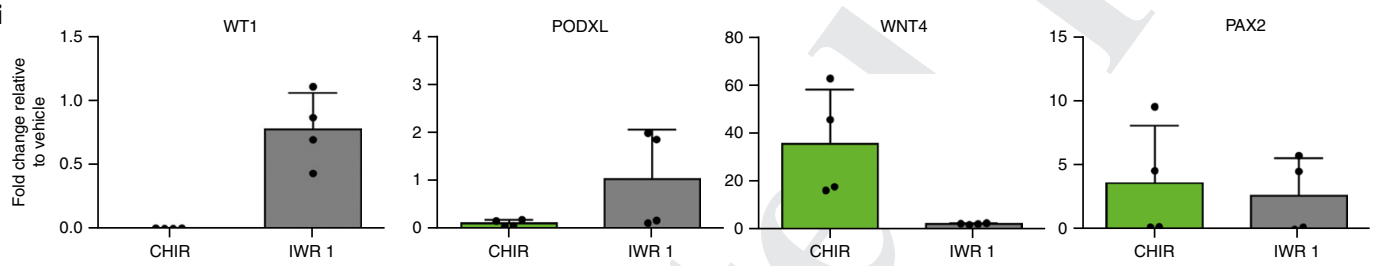

j
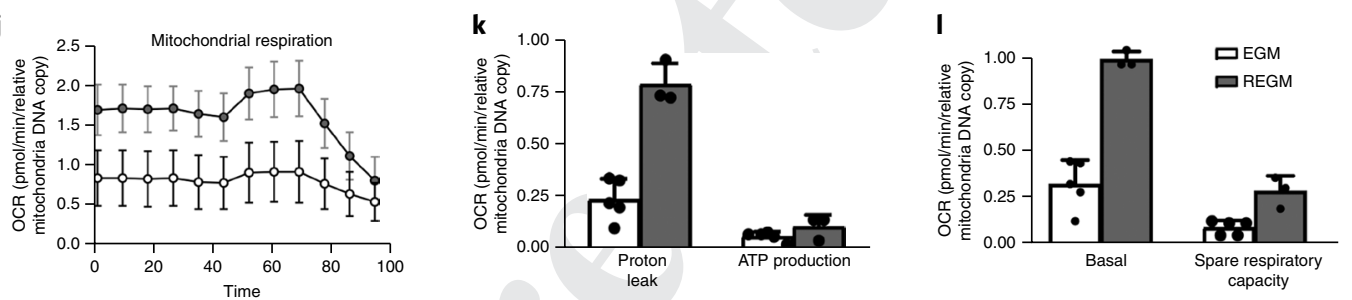

m
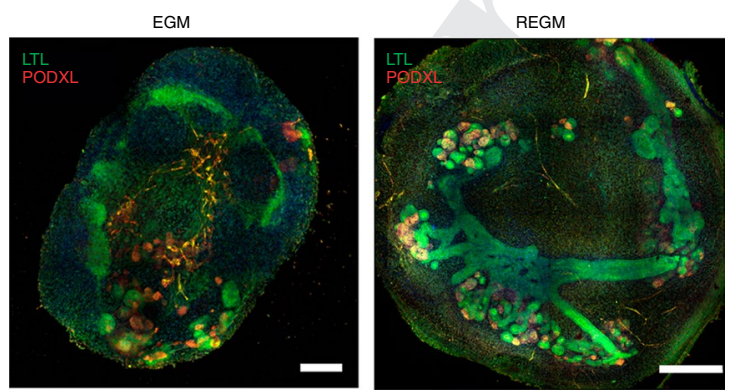

n

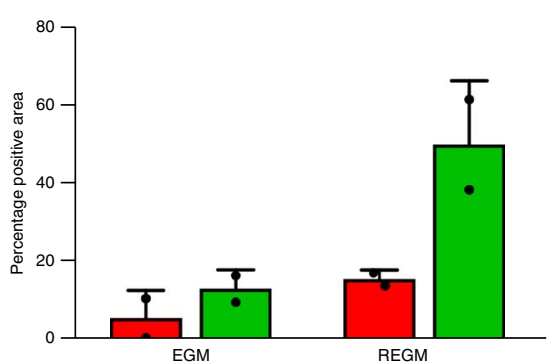

Fig. 2 | Kidney organoids model human kidney organogenesis in vitro. a, Representation of the coculture of day 5 NPCs with mouse embryonic kidney cells. b-d, Bright-field images of reaggregates after $1 \mathrm{~d}(\mathbf{b}), 4 \mathrm{~d}(\mathbf{c})$ and $6 \mathrm{~d}(\mathbf{d})$ in culture. Scale bars, $500 \mu \mathrm{m}$. e, Immunocytochemistry for PAX8, WT1 and HuNu of the reaggregate in d. Scale bar, $250 \mu \mathrm{m}$. $\mathbf{f}$, Magnified views of $\mathbf{d}$. Scale bars, $50 \mu \mathrm{m}$. g-i, Modulation of $\beta$-catenin signalling in kidney organoids with IWR1 and CHIR inhibitors. $\mathbf{g}$, Immunocytochemistry for WT1 and LTL in day 16 kidney organoids with the indicated regimens. Scale bars, $50 \mu$ $\mathrm{m}$. $\mathbf{h}$, Corresponding quantification of the percentage of $\mathrm{WT} 1^{+}$cells and $\mathrm{LTL} \mathrm{L}^{+}$structures. Data are mean $\pm \mathrm{s}$.d. $n=3$ organoids per treatment. One-way analysis of variance with Tukey's post hoc test. For $\% W T 1, F(1.009,2.017)=213.6, P=0.0045$; vehicle versus $C H I R,{ }^{\star \star} P=0.0082 ; C H I R$ versus IWR1, ${ }^{\star \star \star \star} P=0.000034$; vehicle versus IWR1, n.s., not significant, $P=0.9995$. For $\% L T L, F(1.002,2.004)=0.9976, P=0.4232$, not significant. i, Corresponding qPCR analysis (genes are indicated). Data are mean \pm s.d. (three technical replicates). $j-\mathbf{l}$, Energy metabolism profile of kidney organoids maintained in

Q55 EGM or REGM: kinetic oxygen consumption rate (OCR) response (j), inner mitochondrial membrane proton leak and cellular ATP production (k) and basal respiration and spare respiratory capacity (I). Data are normalized to mitochondrial DNA copy number/sample. Data are mean $\pm s . d . n=3(E G M)$ and $n=2$ (REGM) organoids. $\mathbf{m}$, Immunocytochemistry for LTL and PODXL in day 16 kidney organoids under EGM or REGM regimen. Scale bars, $200 \mu \mathrm{m}$ (EGM) and $400 \mu \mathrm{m}$ (REGM). $\mathbf{n}$, Corresponding quantification of the percentage of PODXL + and LTL+ structures. Data are mean \pm s.d. $n=2$ organoids per condition. 


\section{Vascularization of kidney organoids using chick chorioallantoic membrane (CAM)}

Kidney organoids have shown the presence of nascent vascular endothelial cells surrounding renal structures, but lack a proper vascular pattern ${ }^{4,9}$. Of note, only two independent studies have reported in vivo vascularization of either hiPSC-derived NPCs ${ }^{20}$ or kidney organoids ${ }^{21}$ when transplanted under the kidney capsule of immunodeficient mice, identifying in both cases host-derived vascularization. We decided to explore an alternative approach for providing a vascular environment to kidney organoids. We made use of the chick CAM, a highly vascularized extraembryonic tissue that has been used in tumour angiogenesis research ${ }^{22,23}$ and for the grafting of biomaterials ${ }^{24}$. More so than other in vivo models, such as the mouse, CAM represents a naturally immunodeficient environment that offers direct, minimally invasive access to the assay site, thereby facilitating the monitoring of the experiments in situ. We implanted day 16 kidney organoids into the CAM of 7-day-old chick embryos, and then maintained them in ovo for $5 \mathrm{~d}$ (Fig. 3a; Methods). On day 3 of implantation, multiple blood vessels from the CAM were macroscopically distinguished throughout kidney organoids (Fig. 3b, Supplementary Video 1). The circulation of chick blood within kidney organoids was clearly observed after $5 \mathrm{~d}$ (Fig. 3c, Supplementary Video 2). At this stage, in vivo injection of dextran-FITC (fluorescein isothiocyanate) into the CAM allowed for live imaging of the vasculature, confirming the grafting of the organoids into the CAM (Fig. 3d, Supplementary Video 3). Compared with in vitro counterparts (Supplementary Fig. 12a), CAM-implanted kidney organoids (implanted organoids) exhibited glomeruli with an enlarged Bowman's space and tubule-like structures with enlarged lumens (Supplementary Fig. 12b,c, magnified views). Furthermore, CAM blood vessels (indicated with asterisks in Supplementary Fig. 12b,c,d) were found in close vicinity to glomerulus structures. Immunofluorescence analysis of consecutive sections confirmed the presence of chick blood vessels (labelled with Lens culinaris agglutinin) within implanted organoids. The latter were identified by the expression of the human marker $\mathrm{HuNu}$ and the presence of glomerulus-like structures $\left(\mathrm{WT}^{+}{ }^{+}\right.$) (Supplementary Fig. 12e). Next, we tested the ability of implanted organoids to respond to the well known nephrotoxic agent cisplatin. Twenty-four hours after injecting cisplatin into the chick vasculature, levels of KIM-1 (a marker of renal tubule toxicity) and cleaved CASPASE 3 (a classical apoptotic marker) were upregulated in proximal tubular structures $\left(\mathrm{LTL}^{+}\right)$, compared with specimens injected with a control solution (Supplementary Fig. 12f,g).

We next analysed semithin (Fig. 3e) and ultrathin sections (Fig. 3f-k) of implanted organoids. TEM images revealed the presence of aligned podocyte-like cells on one side of a linear basement membrane (Fig. 3f-h). Endothelial-like cells were found on the opposite side of the basal lamina (Fig. 3f) and, occasionally, chicken erythrocytes were observed within the glomerular-like structures (Fig. 3h). Podocyte-like cells exhibited multiple microvilli on the apical surface and extended primary and secondary cell processes on the basal side (Fig. $3 \mathrm{f}-\mathrm{i}$, Supplementary Fig. $12 \mathrm{~h}-\mathrm{j}$ ). Secondary cell processes were bridged by slit diaphragm-like structures (Fig. 3g-i, Supplementary Fig. $12 \mathrm{~h}-\mathrm{j}$ ). These features, which reflect functional differentiation, were not detected in the organoids cultured in vitro (Fig. 1g, h). Furthermore, tubular-like cells with thick brush borders and high mitochondrial content were observed (Fig. 3j,k). Immunofluorescence analysis showed apical localization of PODXL in aligned podocyte-like cells situated on the basement membrane $\left(\mathrm{LAMININ}^{+}\right.$). Conversely, podocin and NEPH1 localized on the podocyte basal side (Fig. 31). Likewise, CD34+ endothelial-like cells (stained with an antihuman specific CD34 antibody) were closely associated with $\mathrm{NEPHRIN}^{+} / \mathrm{PODXL}^{+}$podocyte-like cells within glomerulus-like structures in implanted organoids (Fig. 3m, Supplementary Fig. 12k). Moreover, endothelial-like cells
$\left(\mathrm{CD} 31^{+}\right)$within glomerulus-like structures $\left(\mathrm{PODXL}^{+}\right)$coexpressed the human marker HuNu (Fig. 3n). In contrast, for kidney organoids cultured in vitro, CD34 ${ }^{+}$endothelial-like cells were not found within glomerulus-like structures $\left(\mathrm{PODXL}^{+}\right)$(Supplementary Fig. 12l).

\section{Soft hydrogels enhance the formation of kidney organoids}

Mirroring the exact biochemical (for example, site-specific bioactive ligands) and biophysical (for example, extracellular matrix stiffness, fluid flow, oxygen tension) properties of a physiological environment represents an as yet unaffordable technical approach in tissue engineering. By contrast, fabrication of hydrogels with mechanical properties (for example, Young's modulus) similar to native tissues is a key methodology for guiding cellular responses and differentiation $^{25}$. Therefore, we decided to characterize the specific mechanical properties of the CAM (by measuring the Young's modulus, Supplementary Fig. 13a; Methods), which exhibited a stiffness value of about $1 \mathrm{kPa}$ (Supplementary Fig. 13b), representative of an early embryonic microenvironment ${ }^{26}$ in which undifferentiated cells are primed for lineage commitment ${ }^{27}$. We next explored whether substrates mimicking a soft microenvironment may favour the generation of kidney organoids, compared with stiffer substrates. Thus, we fabricated functionalized polyacrylamide hydrogels of tunable stiffness (ranging from soft, $1 \mathrm{kPa}$, to very rigid, $60 \mathrm{kPa}$ ) as substrates for hPSC differentiation (Supplementary Fig. 14a; Methods). In comparison with rigid hydrogels, hPSCs grown on soft hydrogels under undifferentiated conditions formed tightly compacted hPSC colonies $\left(\mathrm{ECAD}^{+}\right)$(Supplementary Fig. 14b), showing reduced nuclear localization of the mechanotransduction marker Yes-associated protein (YAP) (Supplementary Fig. 14c). RNA-Seq analysis of hPSCs revealed that soft hydrogels promoted the expression of genes related to embryo and mesodermal differentiation (Supplementary Table 3), suggesting that a soft milieu may better replicate early stages of embryonic development, during which time counteracting gene regulatory networks control both pluripotency and differentiation ground states ${ }^{28}$. Based on these observations, we hypothesized that using soft hydrogels during the first steps of monolayer differentiation (including PPS and IM induction) may help guide hPSCs toward renal commitment. PPS induction of hPSCs differentiated on soft hydrogels resulted in higher mRNA levels of $T$ and SALL1 markers when compared with rigid conditions (Supplementary Fig. 15a,b). PPS differentiation was also analysed by RNA-Seq, showing that soft hydrogels induced the expression of genes related to transcription regulation and downregulated genes related to extracellular matrix and basement membrane (Supplementary Table 4, Supplementary Fig. 15c). Induction of PPS-committed cells into IM-committed cells (Supplementary Fig. 16a) showed that soft hydrogels promoted increased mRNA levels of the early IM marker $P A X 2$, the posterior IM marker HOXD11, the anterior IM marker LHX1, and SALL1 when compared with rigid conditions (Supplementary Fig. 16b). Upon differentiation under 3D organotypic culture, IM-committed cells derived on soft hydrogels began to develop RVs one day earlier (at day 7: Supplementary Fig. 16c), and resulted in the generation of more RVs than those derived on rigid hydrogels, as shown by quantitative analysis of PAX2 ${ }^{+}$RVs (Fig. 4a,b). Moreover, day 16 kidney organoids from soft conditions developed more $\mathrm{WT}^{+}$glomeruluslike and $\mathrm{LTL}^{+}$tubule-like structures than those derived from rigid conditions (Fig. 4c,d), and expressed increased mRNA levels of latestage nephron (NPHS1, SCNN1B) and vascularization (ENDOGLIN, VEGFR) markers (Fig. 4e). TEM of day 16 kidney organoids showed the presence of tubule-like structures containing epithelial cells with prominent brush borders in both soft and rigid conditions (Fig. 4f, Supplementary Fig. 17). Interestingly, soft hydrogels induced the differentiation of podocyte-like cells containing slit diaphragmlike structures between the cell processes (Fig. $4 \mathrm{~g}-\mathrm{i}$ ), a podocyte 
a

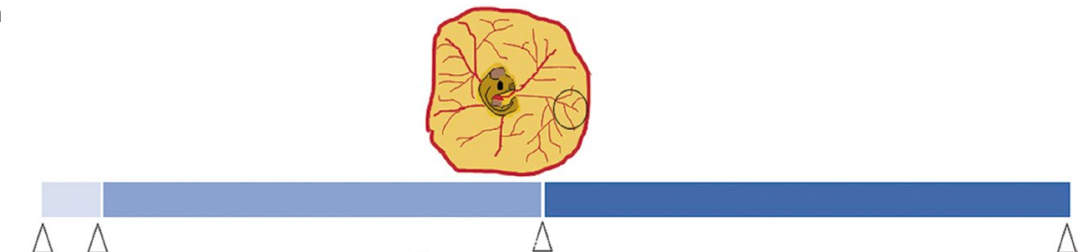
D0 D1 Aspirate albumin
D7 Organoid implantation
D12 Sample collection and analysis
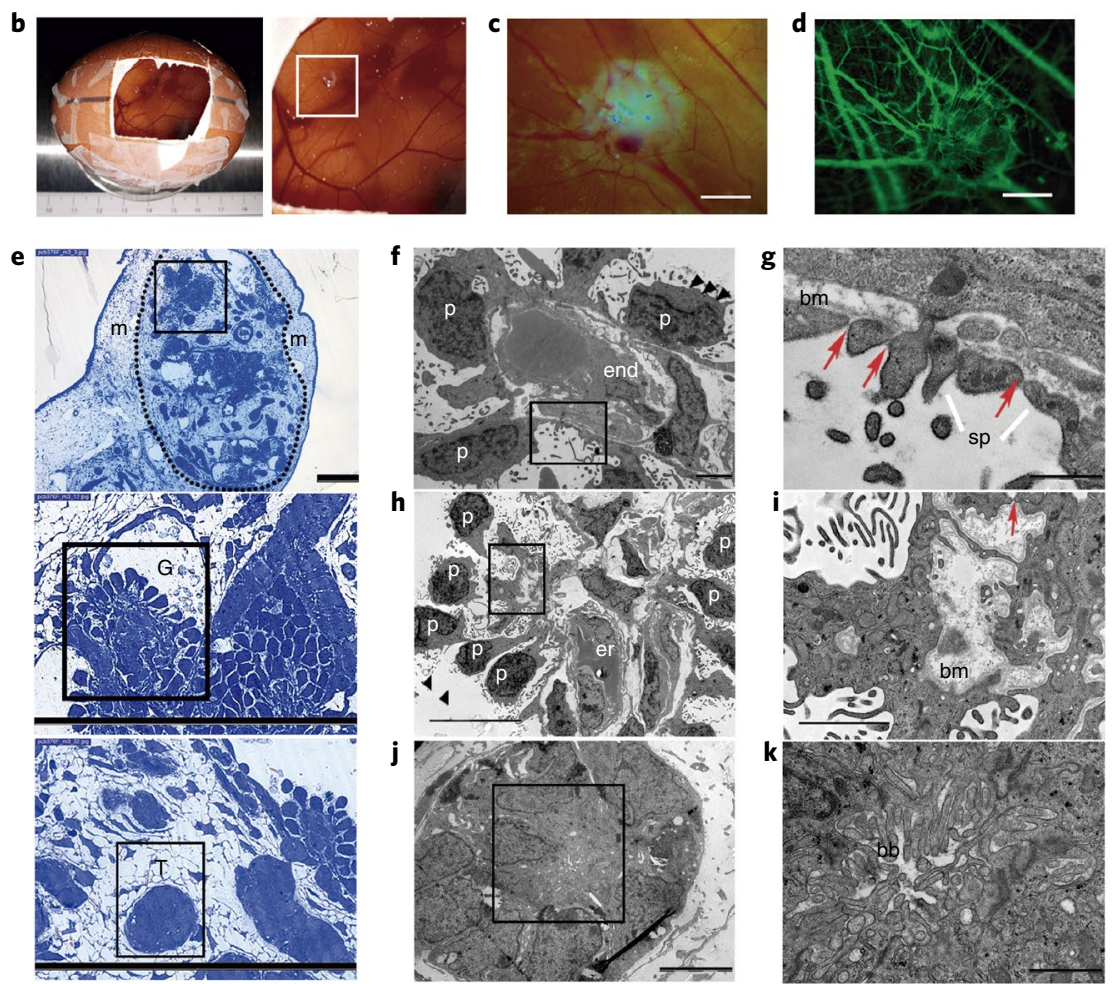

PODXL Laminin

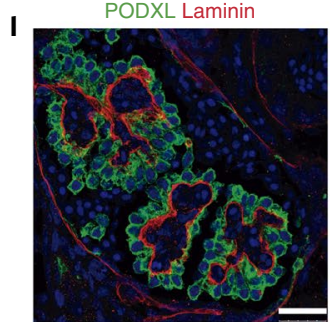

PODXL Podocin

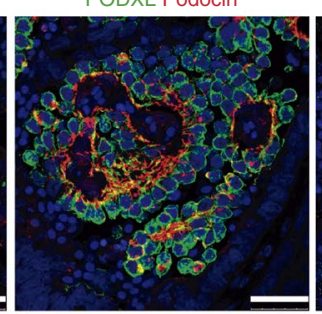

Nephrin Laminin

NEPH1 Laminin
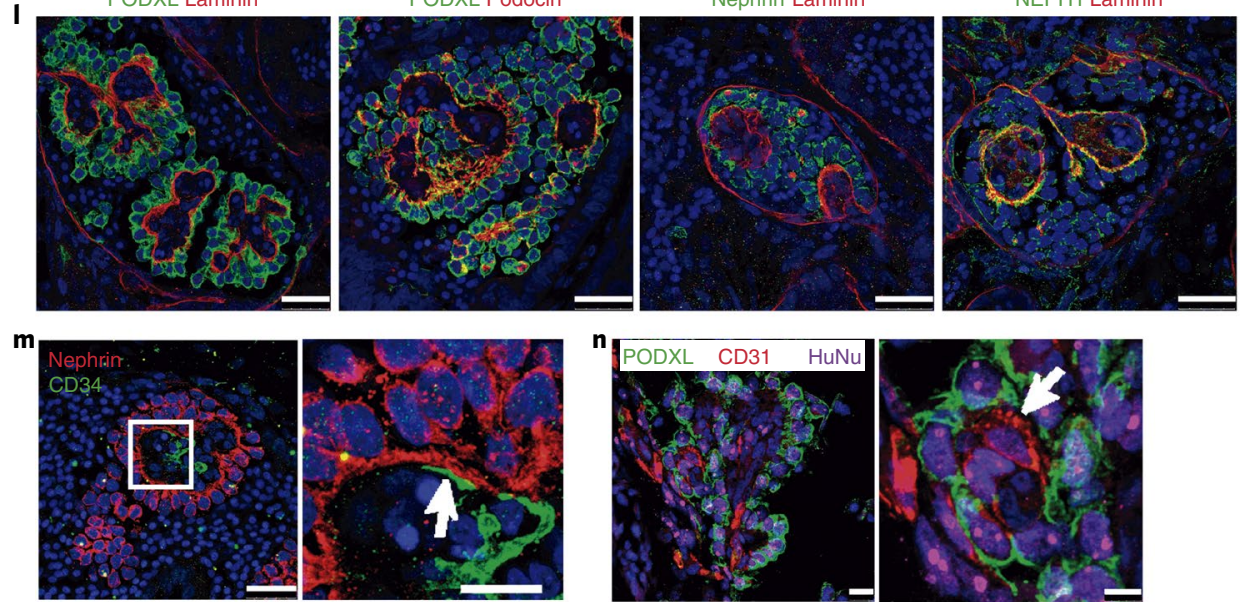

Fig. 3 | In vivo vascularization of kidney organoids using chick CAM. a, Methodology for the implantation of day 16 kidney organoids into chick CAM. $\mathbf{b}, \mathbf{c}$, Macroscopic views of implanted organoids maintained in ovo for $3 \mathrm{~d}(\mathbf{b})$ and $5 \mathrm{~d}(\mathbf{c})$. d , The implanted organoid in $\mathbf{c}$ after intravital injection of dextranFITC through the chick vasculature. Scale bars, $1000 \mu \mathrm{m}(\mathbf{c}, \mathbf{d})$. e, Semithin sections of a kidney organoid (dashed line) implanted in the CAM mesenchyme (m) for $5 \mathrm{~d}$. Magnified views of glomerular (G) and tubular (T) cells are shown. Scale bars, $200 \mu \mathrm{m}, 100 \mu \mathrm{m}$ (magnified views). f-k, TEM of implanted organoids. Magnified views of the boxed regions in $\mathbf{f}, \mathbf{h}, \mathbf{j}$ are shown in $\mathbf{g , i}, \mathbf{k}$, respectively). $\mathbf{f}$, Differentiated podocytes (p) extending primary cell processes

Q7 10 (pp) and apical microvilli (black triangles) are located on one side of the basement membrane (bm) and a vascular endothelial cell (end) is found on the opposite side. $\mathbf{g}$, Slit diaphragm-like structures (red arrows) between secondary cell processes (sp). h, Aligned podocytes showing primary cell processes and apical microvilli. er, chicken erythrocytes. $\mathbf{i}$, A detail of the basement membrane and a slit diaphragm-like structure. $\mathbf{j}$, Tubular-like cells. $\mathbf{k}$, A detail of brush borders (bb). Scale bars, $2 \mu \mathrm{m}(\mathbf{f}), 500 \mathrm{~nm}(\mathbf{g}), 10 \mu \mathrm{m}(\mathbf{h}), 2 \mu \mathrm{m}(\mathbf{i}), 5 \mu \mathrm{m}(\mathbf{j}), 1 \mu \mathrm{m}(\mathbf{k})$. I-n, Confocal microscopy images of glomerular structures in implanted organoids. I, Immunohistochemistry for PODXL, nephrin, NEPH1, podocin and laminin. Scale bars, $25 \mu \mathrm{m}$. $\mathbf{m}$, Immunohistochemistry for nephrin and CD34. Scale bars, $25 \mu \mathrm{m}, 10 \mu \mathrm{m}$ (magnified view). n, Immunohistochemistry for PODXL, CD31 and the human marker HuNu. Scale bars, $10 \mu \mathrm{m}, 5 \mu \mathrm{m}$ (magnified view). White arrows indicate endothelial-like cells in close contact with podocyte-like cells (m,n). 


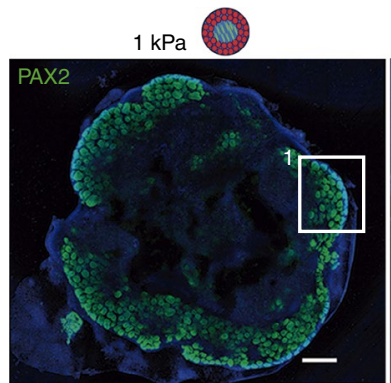

C
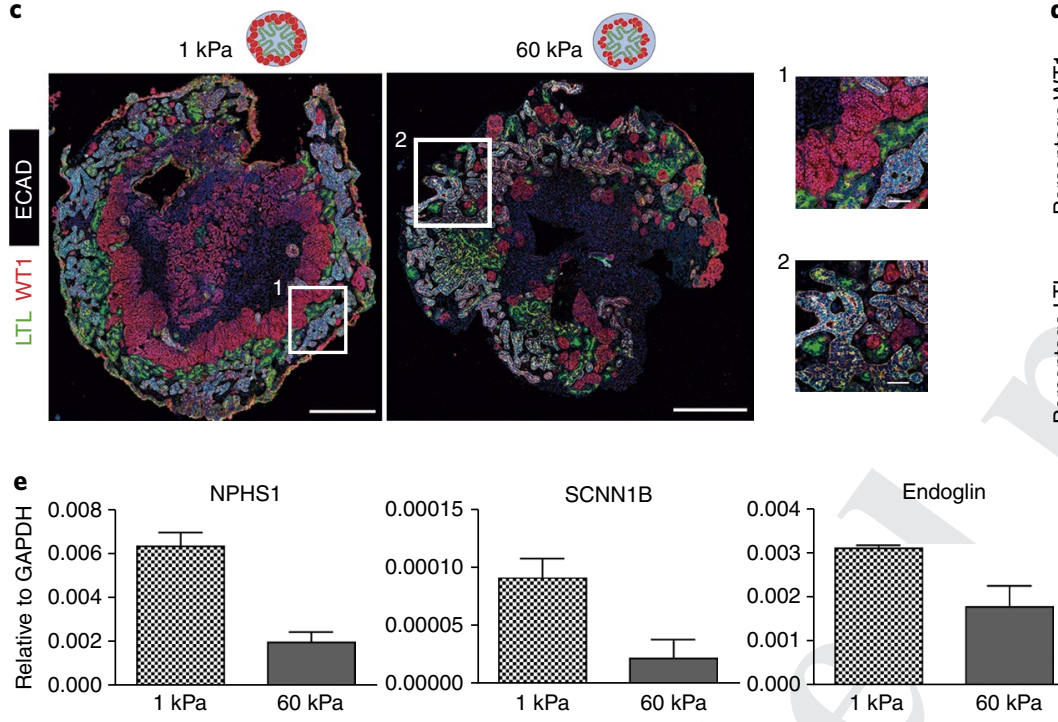

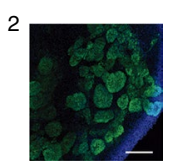

h
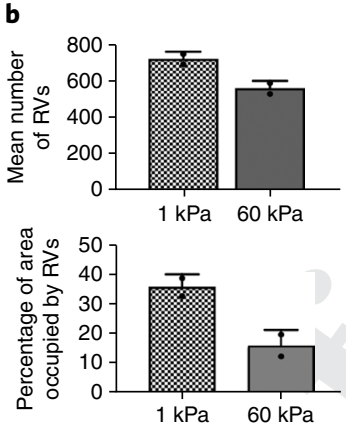

d
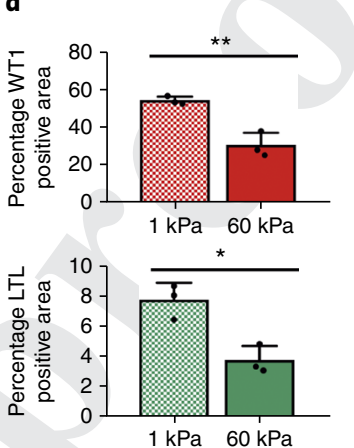

$1 \mathrm{kPa} \quad 60 \mathrm{kPa}$

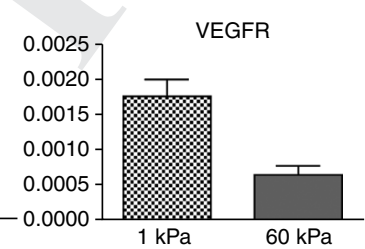

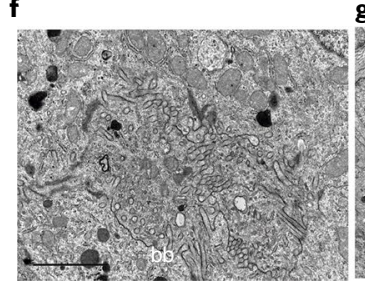

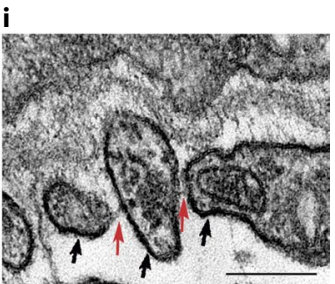

j

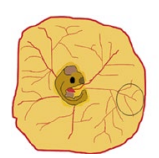

$1 \mathrm{kPa}$

k

5 days implantation Analysis
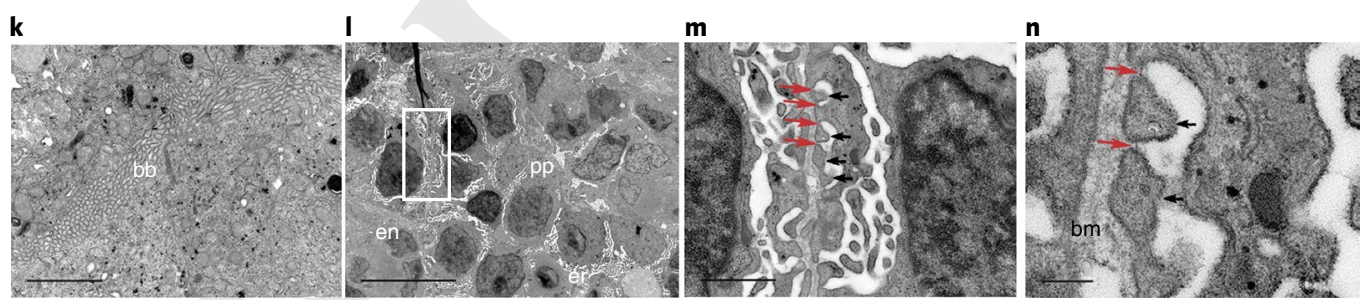

Fig. 4 | Soft hydrogels accelerate the differentiation of kidney organoids. a, Immunocytochemistry for PAX2 in RV-stage organoids generated using $1 \mathrm{kPa}$ or $60 \mathrm{kPa}$ hydrogels. Scale bars, $500 \mu \mathrm{m}, 150 \mu \mathrm{m}$ (magnified views). b, Quantification of $\mathbf{a}$. The mean number of RVs and area percentage occupied by RVs were quantified. Data are mean \pm s.d. $n=2$ organoids per condition. c, Immunohistochemistry for LTL, WT1 and ECAD in day 16 kidney organoids from $1 \mathrm{kPa}$ or $60 \mathrm{kPa}$. Scale bars, $500 \mu \mathrm{m}$ and $50 \mu \mathrm{m}$ (magnified views). d, Quantification of c. The percentages of WT1+ and $\mathrm{LTL}^{+}$area were quantified. Data are mean \pm s.d. $n=3$ organoids per condition. For $W T 1^{+}, t(4)=5.8057,{ }^{\star \star} P=0.0044$. For $L T L^{+} t(4)=4.6023,{ }^{\star} P=0.0100$. Two-tailed Student's $t$-test. e, qPCR analysis of day 16 kidney organoids from $1 \mathrm{kPa}$ or $60 \mathrm{kPa}$ (genes are indicated). Data are mean \pm s.d. (technical replicates). f-i, TEM of day $16 \mathrm{kidney}$ organoids from $1 \mathrm{kPa}$. f, Epithelial tubular-like cells with brush borders (bb). g, Podocyte-like cells with primary cell processes (pp). h,i, Magnified views of g. Secondary cell processes (black arrows) with slit diaphragm-like structures (red arrows). Red asterisks, podocyte membrane protrusions. bm, basement membrane. Scale bars, $2 \mu \mathrm{m}(\mathbf{f}), 5 \mu \mathrm{m}(\mathbf{g}), 500 \mathrm{~nm}(\mathbf{h}), 200 \mathrm{~nm}(\mathbf{i}) . \mathbf{j}$, Day 16 kidney organoids from $1 \mathrm{kPa}$ were implanted into the CAM.

k-n, TEM of implanted kidney organoids from $1 \mathrm{kPa}$. $\mathbf{k}$, Tubular-like cells with brush borders. I, Aligned podocyte-like cells extending primary cell processes near endothelial (en) cells and chicken erythrocytes (er). $\mathbf{m}, \mathbf{n}$, Magnified views of $\mathbf{I}$. Secondary cell processes with slit diaphragm-like structures. Scale bars, $2 \mu \mathrm{m}(\mathbf{k}), 10 \mu \mathrm{m}(\mathbf{I}), 1 \mu \mathrm{m}(\mathbf{m}), 200 \mathrm{~nm}(\mathbf{n})$. 
differentiation feature that was absent in rigid conditions (Fig. 1g,h, Supplementary Fig. 17). Considering these findings, day 16 kidney organoids derived from soft hydrogels were then implanted into the CAM for 5 days (Fig. 4j). TEM revealed the presence of tubular epithelial-like cells with brush borders (Fig. 4k) and numerous glomerular structures containing podocyte-like cells above a dense basement membrane and in close vicinity to endothelial cells and chicken erythrocytes (Fig. 41). Furthermore, induced podocyte-like cells exhibited secondary cell processes with slit diaphragm-like structures (Fig. $4 \mathrm{~m}, \mathrm{n}$ ).

\section{Outlook}

The methodology described here reduced the time needed to generate kidney organoids when compared with previous protocols by about $30 \% \%^{1,3-5,9}$, leading to the generation of kidney organoids that transcriptionally resembled second-trimester human fetal kidneys. This is an improvement over previous findings, in which kidney organoids clustered with trimester 1 human fetal kidneys ${ }^{9}$. Furthermore, here we have shown that kidney organoids implanted into chick CAM successfully engrafted and were vascularized in ovo, providing a straightforward model for nephrotoxicity and kidney disease modelling applications. Importantly, CAM-implanted kidney organoids showed morphological features that reflect functional differentiation compared with in vitro conditions. When CAM stiffness was mimicked in vitro via compliant hydrogels, hPSCs differentiated on soft substrates (CAM-like) generated IM-committed cells that showed an accelerated formation of more RVs and nephron structures than those produced on rigid substrates. Furthermore, kidney organoids generated from soft hydrogels exhibited improved differentiation characteristics when compared with those found under stiffer conditions. These differentiation features were also enhanced after CAM transplantation. Overall, the methodology described here paves the way toward further developing biomimetic approaches that will enhance organoid differentiation (either in vitro or in vivo). These advances will enable future studies of kidney development and disease.

\section{Online content}

Any methods, additional references, Nature Research reporting summaries, source data, statements of data availability and associated accession codes are available at https://doi.org/10.1038/ s41563-019-0287-6.

Received: 5 February 2018; Accepted: 8 January 2019;

\section{References}

1. Taguchi, A. et al. Redefining the in vivo origin of metanephric nephron progenitors enables generation of complex kidney structures from pluripotent stem cells. Cell Stem Cell 14, 53-67 (2014).

2. Lam, A. Q. et al. Rapid and efficient differentiation of human pluripotent stem cells into intermediate mesoderm that forms tubules expressing kidney proximal tubular markers. J Am Soc Nephrol. 25(6), 1211-1225 (2014).

3. Morizane, R. et al. Nephron organoids derived from human pluripotent stem cells model kidney development and injury. Nat. Biotechnol. 33, 1193-1200 (2015).

4. Freedman, B. S. et al. Modelling kidney disease with CRISPR-mutant kidney organoids derived from human pluripotent epiblast spheroids. Nat. Commun. 6, 8715 (2015)

5. Toyohara, T. et al. Cell therapy using human induced pluripotent stem cell-derived tenal progenitors ameliorates acute kidney injury in mice. Stem Cells Transl. Med. 4, 980-992 (2015).

6. Imberti, B. et al. Renal progenitors derived from human iPSCs engraft and restore function in a mouse model of acute kidney injury. Sci. Rep. 5, 8826 (2015).

7. Xia, Y. et al. Directed differentiation of human pluripotent cells to ureteric bud kidney progenitor-like cells. Nat. Cell Biol. 15, 1507-1515 (2013).

8. Takasato, M. et al. Directing human embryonic stem cell differentiation towards a renal lineage generates a self-organizing kidney. Nat. Cell Biol. 16, 118-126 (2014).
9. Takasato, M. et al. Kidney organoids from human iPS cells contain multiple lineages and model human nephrogenesis. Nature 526, 564-568 (2015).

10. Taguchi, A., \& Nishinakamura, R. Higher-order kidney organogenesis from pluripotent stem cells. Cell Stem Cell 21, 730-746 (2017).

11. Musah, S. et al. Mature induced-pluripotent-stem-cell-derived human podocytes reconstitute kidney glomerular-capillary-wall function on a chip. Nat. Biomed. Eng. 1, (2017).

12. Cruz, N. M. et al. Organoid cystogenesis reveals a critical role of microenvironment in human polycystic kidney disease. Nat. Mater. 16, 1112-1119 (2017).

13. Roost, M. S. et al. KeyGenes, a tool to probe tissue differentiation using a human fetal transcriptional atlas. Stem Cell Rep. 4, 1112-1124 (2015).

14. Lindström, N. O. et al. Conserved and divergent features of human and mouse kidney organogenesis. J. Am. Soc. Nephrol. 29(3), 785-805 (2018).

15. Unbekandt, M. \& Davies, J. A. Dissociation of embryonic kidneys followed by reaggregation allows the formation of renal tissues. Kidney Int. 77, 407-416 (2010).

16. Davies, J. A., Unbekandt, M., Ineson, J., Lusis, M. \& Little, M. H. Dissociation of embryonic kidney followed by re-aggregation as a method for chimeric analysis. Methods Mol. Biol. 886, 135-146 (2012).

17. Lindström, N. O. et al. Integrated $\beta$-catenin, BMP, PTEN, and Notch signalling patterns the nephron. eLife 3, e04000 (2014).

18. De Bock, K. et al. Role of PFKFB3-driven glycolysis in vessel sprouting. Cell 154, 651-663 (2013).

19. Narayanan, K. et al. Human embryonic stem cells differentiate into functional renal proximal tubular-like cells. Kidney Int. 83, 593-603 (2013).

20. Sharmin, S. et al. Human induced pluripotent stem cell-derived podocytes mature into vascularized glomeruli upon experimental transplantation. J. Am. Soc. Nephrol. 27(6), 1778-1791 (2016).

21. Van den Berg, C. W. et al. Renal subcapsular transplantation of PSC-derived kidney organoids induces neo-vasculogenesis and significant glomerular and tubular maturation in vivo. Stem Cell Rep. 10, 751-765 (2018).

22. Ribatti, D. Chick embryo chorioallantoic membrane as a useful tool to study angiogenesis. Int. Rev. Cell Mol. Biol. 270, 181-224 (2008).

23. Cimpean, A. M., Ribatti, D. \& Raica, M. The chick embryo chorioallantoic membrane as a model to study tumor metastasis. Angiogenesis 11, 311-319 (2008).

24. Baiguera, S., Macchiarini, P. \& Ribatti, D. Chorioallantoic membrane for in vivo investigation of tissue-engineered construct biocompatibility. J. Biomed. Mater. Res. B 100, 1425-1434 (2012).

25. Vining, K. H. \& Mooney, D. J. Mechanical forces direct stem cell behaviour in development and regeneration. Nat. Rev. Mol. Cell Biol. 18, 728-742 (2017).

26. Przybyla, L., Lakins, J. N. \& Weaver, V. M. Tissue mechanics orchestrate Wnt-dependent human embryonic stem cell differentiation. Cell Stem Cell 19, 462-475 (2016)

27. Ahmed, K. et al. Global chromatin architecture reflects pluripotency and lineage commitment in the early mouse embryo. PLoS One 5, (2010).

28. Theunissen, T. W. \& Jaenisch, R. Mechanisms of gene regulation in human embryos and pluripotent stem cells. Development 144, 4496-4509 (2017).

\section{Acknowledgements}

We are grateful to members of the N. Montserrat laboratory for insightful discussions and critical reading of the manuscript. We thank D. O'Keefe and M. Schwarz for administrative help, L. Bardia, A. Lladó and J. Colombelli from the Advanced Digital Microscopy facility at the Institute for Research in Biomedicine for assistance in confocal microscopy imaging and the Electron Cryo-Microscopy Unit at the Scientific and Technological Centers of the University of Barcelona for their technical assistance. We would particularly like to acknowledge the patients and the Fetal Tissue Bank of Vall d'Hebron University Hospital Biobank (PT13/0010/0021), part of the Spanish National Biobanks Network, for its collaboration. This work has received funding from the European Research Council (ERC) under the European Union's Horizon 2020 research and innovation programme (StG-2014-640525_REGMAMKID to E.G., P.P., C.T. and N.M. and CoG-616480 to X.T.), the European Commission (project H2020FETPROACT-01-2016-731957 to X.T. and P.R.-C.), the Spanish Ministry of Economy and Competitiveness/FEDER (BFU2016-77498-P to L.F. and E.M., BFU2015-65074 to X.T., BFU2016-79916-P to P.R.-C., SAF2015-72617-EXP to N.M., SAF2017-89782-R to N.M. and RYC-2014-16242 to N.M.), the Generalitat de Catalunya and CERCA programme (2014-SGR-927 to X.T. and 2017 SGR 1306 to N.M.), Asociación Española contra el Cáncer (AECC CI2016 to L.F. and E.M., LABAE16006 to N.M.). R.O. is supported by an FI fellowship (Generalitat de Catalunya). P.R.-C. is also supported by Obra Social La Caixa. J.C.I.B. is supported by the G. Harold and Leila Y. Mathers Charitable Foundation, the Leona M. and Harry B. Helmsley Charitable Trust (2012-PG-MED002), the Moxie Foundation, the National Institutes of Health (5R21AG055938), the Universidad Católica San Antonio de Murcia and Fundación Dr. Pedro Guillén. C.H.P. is supported by the Bioengineering Excellence of Scientific Training project, cofunded from the European Union's Horizon 2020 research and innovation programme under the Marie Skłodowska-Curie grant agreement no. 712754 and from the Spanish Ministry of Economy and Competitiveness under the 
Severo Ochoa grant SEV-2014-0425 (2015-2019). N.M. is also supported by CardioCel (TerCel, Instituto de Salud Carlos III). IBEC is the recipient of a Severo Ochoa Award of Excellence from MINECO

\section{Author contributions}

E.G. and N.M. conceived and designed the experiments. E.G., P.P., C.T. and C.H.P. performed the experiments. E.G., P.P., C.T. and R.O. characterized the cell lines and 210 contributed to the protocol design. A.G.-N. and C.H.P. carried out the Seahorse analysis. L.C. contributed to the transcriptomic analysis. E.G., P.P., C.T., R.O., L.F., E.M., D.Z.,

Q111 X.T., P.R.-C., J.M.C., J.C.I.B., C.H.P. and N.M. contributed to data interpretation. E.G. and N.M. wrote the manuscript. All authors commented on the manuscript and contributed to it. N.M. oversaw the project.

\section{Competing interests}

The authors declare no competing interests.

\section{Additional information}

Supplementary information is available for this paper at https://doi.org/10.1038/ s41563-019-0287-6.

Reprints and permissions information is available at www.nature.com/reprints. Correspondence and requests for materials should be addressed to N.M.

Publisher's note: Springer Nature remains neutral with regard to jurisdictional claims in published maps and institutional affiliations.

(๑) The Author(s), under exclusive licence to Springer Nature Limited 2019 


\section{Methods}

Culture of hPSCs. All hPSC lines were obtained after the approval of the Ethics Committee of the Center of Regenerative Medicine in Barcelona and the Comisión de Seguimiento y Control de la Donación de Células y Tejidos Humanos del Instituto de Salud Carlos III (project numbers: 0336E/7564/2016; 0336E/5311/2015; 0336E/15986/2016; 0336E/79489/2015; 00336E/20031/2014). ES [4] hESC and CBiPSsv-4F-40 were obtained from The National Bank of Stem Cells (ISCIII, Madrid). H1 and H9 hESC lines were purchased at Wicell. All the lines were maintained in Essential 8 medium (A1517001, Life Technologies) in cell culture plates coated with $5 \mu \mathrm{g} \mathrm{ml}^{-1}$ vitronectin (A14700, Fisher Scientific) with $5 \% \mathrm{CO}_{2}$ at $37^{\circ} \mathrm{C}$. Cells were passaged every $4-6 \mathrm{~d}$.

hPSC differentiation into renal progenitor cells and generation of 3D kidney organoids. hPSCs grown on vitronectin-coated plates were rinsed twice with PBS (1001-015, Life Technologies) and disaggregated into small cell clusters with $0.5 \mathrm{mM}$ EDTA (E9884, Sigma). Cells were then seeded onto vitronectincoated culture plates at a density of $5 \times 10^{4}-1.5 \times 10^{4}$ cells per $\mathrm{cm}^{2}$ in Essential 8 medium (day -5). After overnight culture, the differentiation was initiated by treating hPSCs with $8 \mu$ M CHIR (SML1046, Sigma) in advanced RPMI 1640 basal medium (12633-012, Life Technologies) supplemented with $2 \mathrm{mM}$ L-GlutaMAX (35050-038, Life Technologies) and penicillin/streptomycin (penicillin $10,000 \mathrm{U} \mathrm{ml}^{-1}$ :streptomycin $10,000 \mu \mathrm{g} \mathrm{ml}^{-1} ; 15140122$, Life Technologies) for $3 \mathrm{~d}$ (from day -4 to day -1 ). Next, cultures were treated with $200 \mathrm{ng} \mathrm{ml}^{-1}$ FGF9 (10023B, Peprotech), $1 \mu \mathrm{ml}^{-1}$ heparin (H3149-10KU, Sigma) and $10 \mathrm{ng} \mathrm{ml}^{-1}$ activin A (338-AC-050, Vitro) for $1 \mathrm{~d}$ (from day -1 to day 0 ). Media changes were performed every day. On day 0 , single-cell suspensions were obtained by dissociating cells with Accumax (07921, Stem Cell Technologies). Cells were then resuspended in advanced RPMI 1640 basal medium containing $3 \mu \mathrm{M} \mathrm{CHIR,} 200 \mathrm{ng} \mathrm{ml}^{-1} \mathrm{FGF} 9$ and $1 \mu \mathrm{g} \mathrm{ml}^{-1}$ heparin, placed in 96-well plates (V bottom) at $5 \times 10^{5}$ cells per well, spun down ( $300 \mathrm{~g}$ for $3 \mathrm{~min}$ ) and maintained in culture for $2 \mathrm{~d}$ without medium change. On day 2, cell spheroids were placed onto Transwells (CLS3460, Sigma) and cultured in advanced RPMI 1640 basal medium containing $3 \mu \mathrm{M}$ CHIR, $200 \mathrm{ng} \mathrm{ml}^{-1}$ FGF9 and $1 \mu \mathrm{g} \mathrm{ml}^{-1}$ heparin for another $1 \mathrm{~d}$. On day 3 , CHIR was removed and organoids were maintained in advanced RPMI 1640 basal medium with $200 \mathrm{ng} \mathrm{ml}^{-1} \mathrm{FGF} 9$ and $1 \mu \mathrm{g} \mathrm{ml}^{-1}$ heparin for another $4 \mathrm{~d}$. From day 7 organoids were maintained in advanced RPMI 1640 basal medium until day 16 unless otherwise indicated, changing the medium every second day.

Immunocytochemistry. After a single wash with PBS, samples were fixed with $4 \%$ paraformaldehyde (153799, Aname) for $20 \mathrm{~min}$ at room temperature. Next, samples

were washed twice with PBS and further blocked using Tris-buffered saline (TBS) with $6 \%$ donkey serum (S30, Millipore) and $1 \%$ Triton X -100 (T8787, Sigma) for $1 \mathrm{~h}$ at room temperature. Samples were then treated overnight at $4^{\circ} \mathrm{C}$ with primary antibodies diluted in antibody dilution buffer consisting of TBS with $6 \%$ donkey serum and $0.5 \%$ Triton X-100. After three rinses with antibody dilution buffer, samples were treated for $4 \mathrm{~h}$ at room temperature with fluorescent-conjugated secondary antibodies (Alexa Fluor (A) 488-, Cy3- or A647-; 1:200). A previous blocking step with a streptavidin/biotin blocking kit (SP-2002, Vector Labs) was performed when samples were assayed for biotinylated LTL (B-1325, Vector Labs) and Alexa Fluor 488-conjugated streptavidin (SA5488, Vector Labs) was used to detect $\mathrm{LTL}^{+}$cells. Nuclei were detected using 4,6-diamidino-2-phenylindole (DAPI; 1:5000, D1306, Life Technologies) for $30 \mathrm{~min}$. For mounting, samples were immersed in Fluoromount-G (0100-01, Southern Biotech). Image acquisition was carried out using an SP5 Leica microscope or a Zeiss LSM780 confocal microscope. Primary antibodies and associated information are provided in Supplementary Table 5.

Electron microscopy. After fixation of samples with $2.5 \%$ glutaraldehyde containing $1 \%$ tannic acid in $0.1 \mathrm{M}$ phosphate buffer ( $\mathrm{pH} 7.4$ ), samples were postfixed for $1 \mathrm{~h}$ at $4{ }^{\circ} \mathrm{C}$ with $1 \% \mathrm{OsO}_{4}$ in $0.1 \mathrm{M}$ phosphate buffer. Graded ethanol series were performed followed by epoxy resin embedding. Toluidine blue staining was performed in semithin sections before examination using a light microscope. Then, ultrathin sections were obtained using an EM UC7 ultramicrotome (Leica Microsystems) and collected on copper grids. $4 \%$ uranyl acetate and lead citrate were then used for staining. Samples were subsequently analysed with a JEM 1230 electron microscope (JEOL).

Total RNA isolation and qPCR with reverse transcription. TRI Reagent was employed for total RNA purification following the manufacturer's recommendations (T9424, Sigma). TURBO DNase inhibitor (AM1907, Ambion) was used in order to eliminate any residual genomic DNA. Complementary DNA was synthesized from $1 \mu \mathrm{g}$ of RNA using a Cloned AMV First-Strand cDNA synthesis kit (12328, Invitrogen). Quantitative PCR with reverse transcription (QUANTSTUDIO 5 Applied Biosystems, Thermo Fisher Scientific) was used to quantify gene expression from cDNAs ( $25 \mathrm{ng} /$ well) using PowerUp Sybr Green Master Mix (A25742, Thermo Fisher Scientific). GAPDH or Rplp0 were used for data normalization. Primer sequences used in this study are listed in Supplementary Table 6 .

Next-generation RNA sequencing. Sequencing libraries were prepared from $1 \mu$ $\mathrm{g}$ of total RNA (previously isolated using TRI Reagent-T9424, Sigma) using an
Illumina TruSeq RNA Sample Prep Kit (catalogue no. FC-122-1001). Sequencing was carried out to produce between 50 and 60 million paired end reads/sample using the Illumina Hiseq 2500 platform. Raw sequences were inspected for their quality using FastQC (version v0.11.5) ${ }^{29}$ and trimmed using Skewer (version $0.2 .2)^{30}$. STAR mapper (version 2.5.3a) ${ }^{31}$ was used to align the data to the human reference genome (GRCh38). The option '-quantMode' was employed to count the number of mapped tags within genes (annotation Gencode v26). On average, $90 \%$ of tags were univocally mapped either to the genomic or to a splice junction. Data from Chuva de Sousa Lopes (SRP055513) ${ }^{13}$ were analysed in the same way. Read counts per genes were finally analysed using the R statistical package DESeq $2^{32}$. Since the two experiments were performed using different techniques (RNA-Seq and DeepSAGE), we used the ComBat function from the svaseq R package ${ }^{33}$ on rlog-transformed read counts in order to mitigate the batch effect. Scaled rlog values were then used to calculate the sample-to-sample distance and plotted as a dendrogram. Data from Little (SRP059518) ${ }^{9}$ and McMahon (SRP111183) ${ }^{14}$ were analysed using the same procedure. The package Keygenes ${ }^{13}$ was used to classify every sample according to its similarity to a tissue. Keygenes compares the transcriptional profiles of test samples with that from organs or cell types from a training set. In this study, the 'fetal wo' training set was used. This training set contains transcriptional data from 17 fetal organs.

Flow cytometry. Cells were dissociated using Accumax (07921, Stem Cell Technologies) for $5 \mathrm{~min}$ at $37^{\circ} \mathrm{C}$. Next, cells were resuspended in PBS and incubated with LIVE/DEAD Fixable Violet stain reagent (L23105, Life Technologies) (1:1000) for $30 \mathrm{~min}$ in the dark. For intracellular staining, a Foxp3/ Transcription Factor Staining Buffer Set (00-5523-00, Labclinics) was used according to manufacturer's instructions. Briefly, cell suspensions were fixed in the dark for 30-60 min at room temperature with Foxp3 fixation/permeabilization working solution. Permeabilization of samples was performed using the permeabiljzation buffer for $5 \mathrm{~min}$ at room temperature. Blocking was performed using $2 \%$ fetal bovine serum for $15 \mathrm{~min}$. Incubations with conjugated antibodies were performed for $30 \mathrm{~min}$. The antibodies used were OCT4 conjugated to Alexa Fluor 488 (560253, BD Pharmigen), brachyury conjugated to allophycocyanin (IC2085A, R\&D Systems) and PAX2 (AF3364, R\&D Systems) conjugated to A488 using a Lightning-Link ${ }^{\circ}$ Rapid conjugation kit (322-0010, Innova Biosciences) following the manufacturer's instructions. Samples were then washed with permeabilization buffer and resuspended in PBS $+2 \%$ fetal bovine serum. For cell sorting experiments, kidney organoids were stained with fluoresceinconjugated LTL (FL-1321, Vector Laboratories) as described elsewhere ${ }^{12}$. Kidney organoids were then dissociated to single cells using Accumax (07921, Stem Cell Technologies) for $15 \mathrm{~min}$ followed by $0.25 \%$ (wt/vol) trypsin (25300-054, Life Technologies) for $15 \mathrm{~min}$ at $37^{\circ} \mathrm{C}$. SA3800 software version 2.0.4 (SONY) was used to acquire flow cytometry samples in the Sony SA3800 spectral cell analyser (SONY). FACSDiva software version 8.0.1 (BD Biosciences) was used in the FACS Aria Fusion instrument (BD Biosciences) for cell sorting experiments. FlowJo software version 10 was used to analyse the data.

Reaggregation of mouse embryonic kidney cells with hPSC-derived NPCs. These experiments were performed following approval by the Ethics Committee on Animal Research of the University of Barcelona, Spain (protocol no. OB 391/18). Reaggregation experiments were carried out as previously described in ref. ${ }^{16}$. In brief, embryonic kidneys from 11.5-12.5 d post conception were collected from time-mated pregnant C57BL/6J mice. Kidney rudiments were removed from mouse embryos by manual dissection under a dissecting microscope. Dissociation of kidney rudiments into single cells was performed by incubating kidneys with $0.25 \%$ (wt/vol) trypsin (25300-054, Life Technologies) for $1-2 \mathrm{~min}$ at $37^{\circ} \mathrm{C}$, followed by quenching of trypsin using complete medium (MEM $+10 \%$ fetal bovine serum + penicillin/streptomycin) and pipetting up and down vigorously for $30 \mathrm{~s}$ to disaggregate kidneys. Day 5 organoids were dissociated into single cells with Accumax (07921, Stem Cell Technologies). The resultant cell suspensions were sieved through a $40 \mu \mathrm{m}$ pore cell strainer. Next, $7.2 \times 10^{5}$ mouse kidney cells were combined with $8 \times 10^{3}$ cells from day 5 organoids, placed in a 96-well plate (V bottom) in complete medium with $10 \mu \mathrm{M}$ ROCK inhibitor (72304, Stem Cell Technologies), spun down ( $300 \mathrm{~g}$ for $3 \mathrm{~min}$ ) and incubated at $37^{\circ} \mathrm{C}, 5 \% \mathrm{CO}_{2}$ to allow aggregate formation. After overnight culture, aggregates were placed onto Transwells (CLS3460, Sigma) and maintained in complete medium at $37^{\circ} \mathrm{C}$ and $5 \% \mathrm{CO}_{2}$ for $4-6 \mathrm{~d}$. Medium changes were performed every $48 \mathrm{~h}$.

Nephron patterning assays. For nephron patterning assays, samples were cultured in basic differentiation media supplemented with $10 \mu \mathrm{M}$ DAPT (565770, Sigma), $3 \mu$ M CHIR (SML1046, Sigma) or $5 \mu$ M IWR1 (681669, Sigma) from day 8 to day 16. Organoids were then collected for RNA isolation and fixed with $4 \%$ paraformaldehyde for immunocytochemistry.

Seahorse analysis. Kidney organoids at day 16 of differentiation were resuspended in warm Seahorse XF Assay Medium (Seahorse Bioscience). Individual organoids were transferred to an islet plate (one organoid per well) containing $400 \mu \mathrm{l}$ of medium per well. After $1 \mathrm{~h}$ of incubation at $37^{\circ} \mathrm{C}$, plates were loaded into an XF24 respirometry machine (Seahorse Bioscience). Uncoupled and maximum OCR were 
assayed with oligomycin $(1 \mu \mathrm{M})$ and FCCP $(1.5 \mu \mathrm{M})$. To inhibit complex I- and III-dependent respiration, rotenone $(1 \mu \mathrm{M})$ and antimycin $\mathrm{A}(1 \mu \mathrm{M})$ were used, respectively. OCR represents the oxygen tension and acidification of the medium as a function of time $(\mathrm{pmol} \mathrm{min}-1)$.

Implantation of kidney organoids onto chick CAM. Following animal care guidelines in Spain, no approval was required to perform the experiments described here. Briefly, fertilized white Leghorn chicken eggs were supplied by Granja Gibert. Eggs were placed horizontally in a humidified atmosphere at $38^{\circ} \mathrm{C}$ in a Javier Masalles $240 \mathrm{~N}$ incubator. After $24 \mathrm{~h}, 3 \mathrm{ml}$ of albumin was evacuated from the egg using a 18-gauge syringe. At embryonic day 7 (ED 7), a small window was created by cutting the egg shell using a sterile scalpel. Then, day 16 kidney organoids were implanted onto the surface of the CAM (one kidney organoid per egg) by gently scraping the upper CAM layer (avoiding bleeding or visible rupture of capillaries) at the desired implantation site. Egg windows were sealed with conventional plastic tape and incubated for 3-5 additional days (until ED 10-12).

Intravital imaging of the CAM vasculature. Under a dissecting microscope, superficial CAM veins were injected with $1 \mathrm{mg} \mathrm{ml}^{-1}$ FITC-dextran (2 MDa) (FD2000S, Sigma) in PBS using a 30-gauge Hamilton syringe, allowing solutions to circulate for $5 \mathrm{~min}$. Injected volumes were kept at $50 \mu \mathrm{l}$. Live imaging was performed using a MZ10 F Leica stereomicroscope equipped with a MC170 HD Leica camera.

Nephrotoxicity assay. Chick embryos (ED 14) that contained kidney organoids implanted into the CAM were intravenously injected with desired dosages of cisplatin (P4394, Sigma) using a 30-gauge Hamilton syringe, as previously reported $^{34}$. Specimens injected with control solution (without cisplatin) were used as controls. Eggs were then sealed with conventional plastic tape and incubated overnight at $38^{\circ} \mathrm{C}$. After $24 \mathrm{~h}, \mathrm{CAM}$-implanted kidney organoids were collected and analysed.

Histological analysis and immunohistochemistry on CAM-implanted kidney organoids. CAM-implanted kidney organoids were harvested at day 5 of the implantation period, fixed in $4 \%$ paraformaldehyde at $4{ }^{\circ} \mathrm{C}$ overnight and embedded in paraffin. For histological analysis, $5 \mu \mathrm{m}$ thick sections were stained with haematoxylin and eosin. For immunohistochemistry, antigen retrieval consisting of citrate buffer ( $\mathrm{pH} 6$ ) at $95^{\circ} \mathrm{C}$ for $30 \mathrm{~min}$ was performed. Samples were then blocked with TBS containing 3\% donkey serum and 1\% Triton X-100 for $1 \mathrm{~h}$ at room temperature. Subsequently, primary antibodies were used overnight at $4{ }^{\circ} \mathrm{C}$ in TBS with $3 \%$ donkey serum and $0.5 \%$ Triton X-100. After three washing steps with TBS containing 3\% donkey serum and $0.5 \%$ Triton X-100, samples were treated with the appropriate conjugated secondary antibodies (Alexa Fluor 488-, Cy3- or A647-; all 1:200) for $2 \mathrm{~h}$ at room temperature. Nuclei were stained with DAPI (1:5000, D1306, Life Technologies) for $10 \mathrm{~min}$. Samples were immersed in Fluoromount-G (0100-01, Southern Biotech). Image acquisition was carried out using a SP5 Leica microscope or a Zeiss LSM780 confocal microscope. Primary antibodies and associated information are provided in Supplementary Table 5.

Determination of the Young's modulus of the chick CAM. The ball indentation method was used to assess the Young's modulus $(E)$ of the chick CAM, as described in ref. ${ }^{35}$. The indentation depth $(d)$ was calculated based on the derivative of fluorescence intensity profile using a custom-made MATLAB code. $E$ was calculated from the indentation force $(F), d$ and the radius of the ball indentor $(R)$. For $d<0.3 R$, the Hertz contact mechanics model was used to calculate $E$ as follows: $E=\left[3\left(1-^{2}\right) F\right] / 4 R^{0.5} d^{1.5}$, where $\nu$ is the Poisson's ratio of the CAM.

Fabrication of functionalized polyacrylamide hydrogels. Glass-bottom dishes were loaded with a solution of acetic acid, 3-(trimethoxysilyl)propyl methacrylate (Sigma) and ethanol (1/1/14). Wells were next rinsed three times with $96 \%$ ethanol. Different concentrations of acrylamide and bis-acrylamide were combined with a solution containing $0.5 \%$ ammonium persulfate, $0.05 \%$ tetramethylethylenediamine (Sigma) and $2 \%$ fluorescent $200 \mathrm{~nm}$ far-red carboxylated nanobeads (Invitrogen). Specifically, concentrations of 5 and $0.04 \%$ of acrylamide and bis-acrylamide were used for the softer hydrogels, and 12\% and $0.25 \%$ of acrylamide and bis-acrylamide for the stiffer hydrogels, resulting in a nominal Young's modulus of $1 \mathrm{kPa}$ and $60 \mathrm{kPa}$, respectively, according to ref. ${ }^{36}$. The substrates were functionalized as previously described in ref. ${ }^{37}$. Briefly, a drop containing $1 \mathrm{mg} \mathrm{ml}^{-1}$ acrylic acid NHS (A8060, Sigma), $0.2 \%$ bis-acrylamide, $0.2 \%$ tetramethacrylate (408360, Sigma) and $0.05 \%(\mathrm{w} / \mathrm{v})$ Irgacure 2959 was added on the surface of the hydrogel and photoactivated under exposure to ultraviolet light for $10 \mathrm{~min}$. Afterwards, functionalized hydrogels were washed with HEPES and PBS and incubated overnight with $50 \mu \mathrm{g} \mathrm{ml}^{-1}$ vitronectin (A14700, Fisher Scientific) at $4^{\circ} \mathrm{C}$.

Quantification of immunofluorescence images. A custom-made MATLAB code was used to perform the quantification of immunofluorescence images. For quantification of number and area of RVs, the DAPI image was first smoothed with a mean filter to homogenize the intensity values of the nuclei within an RV.
This image was converted into a binary image after applying an intensity threshold. The binary image was used to segment the RVs by applying a watershed algorithm. From this segmentation, a list containing the area of each RV and the number of RVs was obtained. The entire area of the organoid was identified using the same principle but reducing the threshold for the binary conversion. The percentage of area occupied by RVs was calculated by adding the area of all RVs identified divided by the entire area of the organoid. For quantification of $\mathrm{LTL}^{+}$and $\mathrm{PODXL}^{+}$ structures, the percentage of area occupied by $\mathrm{LTL}^{+}$and $\mathrm{PODXL}^{+}$structures was calculated using the same procedure as described above. For quantification of $\mathrm{WT}^{+}$cells, the DAPI image was converted into a binary image after applying an intensity threshold. The binary image was used to segment the nuclei by applying a watershed algorithm. All nuclei found were counted. The same procedure was used to identify $\mathrm{WT}^{+}{ }^{+}$nuclei. The positive nuclei in WT1 images were divided by the number of DAPI nuclei, giving a percentage of $\mathrm{WT}^{+}$cells for each condition.

Human kidney material. Primary human proximal tubular cells were obtained from collaborators at Hospital Clinic de Barcelona, Spain. The procedure was approved by the ethics committee of Hospital Clinic de Barcelona (project no. 2009/5023). Primary renal proximal tubular epithelial cells were obtained in the laboratory of origin as previously described ${ }^{38}$. Human fetal kidney samples included in this study were provided by the Fetal Tissue Bank of Vall d'Hebron University Hospital Biobank (PT13/0010/0021), part of the Spanish National Biobanks Network, and they were processed following standard operating procedures with the appropriate approval of the Ethical and Scientific Committees (project no. 0336E/9934/2015). The gestational age of human fetal kidney samples was determined using ultrasound heel-to-toe and crown-to-rump measurements ${ }^{39}$. Human fetal kidney samples from 13,16 and 22 weeks of gestation were supplied as whole tissues embedded in OCT (at $-80^{\circ} \mathrm{C}$ ) and as frozen tissue samples for RNA extraction.

Statistics and reproducibility. Data are mean \pm standard deviation (s.d.). Statistical differences between two groups were tested with a two-tailed Student's t-test or one-way analysis of variance followed by Tukey's post hoc test. Data were statistically significant if $P<0.05$. Number of replicates $(n=x), P$ values and degrees of freedom are included in the figure legends. GraphPad Prism version 6.01 software was used for statistical analysis. A table summarizing sample size, number of experiments and statistical test results (when applicable) for each figure panel is also provided as Supplementary Table 7.

For in vitro experiments, two to six organoids were analysed at the times and conditions indicated in each experiment. For ex vivo reaggregation assay, we used one to three pregnant mice (to collect embryonic kidneys from mouse embryos) per experiment. Two or three reaggregates were analysed per experiment. For implantation of organoids into chick CAM in ovo, about 6-22 chicken eggs were used per experiment and about 2-10 implanted kidney organoids were analysed per experiment.

RNA-Seq of kidney organoids during the time course differentiation was performed on six pooled kidney organoids at each time analysed from two independent experiments (Fig. 1c and Supplementary Fig. 9).

For main figures where representative immunofluorescence images are shown, at least $n=2$ biologically independent kidney organoids were analysed from independent experiments showing similar results (Fig. 1b, $n=2$ organoids; Fig. 2 g, $n=3$ organoids per treatment; Fig. $2 \mathrm{~m}, n=4$ organoids per culture condition; Fig. 31,m, $n=2$ implanted organoids; Fig. $3 \mathrm{n}, n=3$ implanted organoids; Fig. 4a, $n=2$ organoids per stiffness condition; Fig. $4 \mathrm{c}, n=3$ organoids per stiffness condition).

For main figures where representative TEM images are shown, $n=2$ biologically independent kidney organoids were analysed from independent experiments showing similar results (Fig. 1e-j, Fig. 3e-k, Fig. 4f-i and Fig. 4k-n).

Macroscopic images of kidney organoids after implantation into chick CAM are representative of three independent CAM implantation experiments (Fig. 3b, $n=6$ implanted organoids; Fig. 3 c, $n=10$ implanted organoids; Fig. 3 d, $n=3$ implanted organoids after dextran-FITC injection).

For supplementary figures, complete information on the number of independent experiments and samples analysed is provided in the corresponding figure legends.

Reporting Summary. Further information on research design is available in the Nature Research Reporting Summary linked to this article.

\section{Code availability}

MATLAB codes can be requested from the corresponding author.

\section{Data availability}

RNA-Seq data are publicly available in Gene Expression Omnibus (GEO, http:// www.ncbi.nlm.nih.gov/geo) under the accession numbers GSE108349, GSE108350 and GSE108351. All remaining datasets supporting the findings described here are available within the article and its supplementary information files. Additionally, data are available from the corresponding author upon reasonable request. 


\section{References}

29. Andrews, S. FastQC: a quality control tool for high throughput sequence data. http://www.bioinformatics.babraham.ac.uk/projects/fastqc (2010).

30. Jiang, H., \& Lei, R. \& Ding, S. W. \& Zhu, S. Skewer: A fast and accurate adapter trimmer for next-generation sequencing paired-end reads. BMC Bioinform. 15, 182 (2014).

31. Dobin, A. et al. STAR: ultrafast universal RNA-seq aligner. Bioinformatics 29(1), 15-21 (2013).

32. Love, M. I., \& Huber, W. \& Anders, S. Moderated estimation of fold change and dispersion for RNA-seq data with DESeq2. Genome Biol. 15(12), 550 (2014).

33. Leek, J. T. Svaseq: removing batch effects and other unwanted noise from sequencing data. Nucl. Acids Res. 42, e161 (2014).

34. Kue, C. S., Tan, K. Y., Lam, M. L. \& Lee, H. B. Chick embryo chorioallantoic membrane (CAM): an alternative predictive model in acute toxicological studies for anti-cancer drugs. Exp. Anim. 64, 129-138 (2015).
35. Lee, D., Rahman, M. M., Zhou, Y. \& Ryu, S. Three-dimensional confocal microscopy indentation method for hydrogel elasticity measurement. Langmuir 31, 9684-9693 (2015).

36. Yeung, T. et al. Effects of substrate stiffness on cell morphology, cytoskeletal structure, and adhesion. Cell Motil. Cytoskelet. 60, 24-34 (2005).

37. Przybyla, L., Lakins, J. N., Sunyer, R., Trepat, X. \& Weaver, V. M. Monitoring developmental force distributions in reconstituted embryonic epithelia. Methods 94, 101-113 (2016).

38. Montserrat, N. et al. Generation of induced pluripotent stem cells from human renal proximal tubular cells with only two transcription factors, OCT4 and SOX2. J. Biol. Chem. 287, 24131-24138 (2012).

39. O'Rahilly, R. \& Müller, F. Developmental stages in human embryos: revised and new measurements. Cells Tissues Organs 192, 73-84 (2010). 


\section{QUERY FORM}

\begin{tabular}{|l|c|}
\hline \multicolumn{2}{|c|}{ Nature Materials } \\
\hline Manuscript ID & [Art. Id: 287] \\
\hline Author & Elena Garreta \\
\hline
\end{tabular}

\section{AUTHOR:}

The following queries have arisen during the editing of your manuscript. Please answer by making the requisite corrections directly in the e.proofing tool rather than marking them up on the PDF. This will ensure that your corrections are incorporated accurately and that your paper is published as quickly as possible.

\begin{tabular}{|c|c|}
\hline Query No. & Nature of Query \\
\hline Q1: & $\begin{array}{l}\text { Please check your article carefully, coordinate with any co-authors and enter all final edits clearly in the eproof, } \\
\text { remembering to save frequently. Once corrections are submitted, we cannot routinely make further changes to } \\
\text { the article. }\end{array}$ \\
\hline Q2: & $\begin{array}{l}\text { Note that the eproof should be amended in only one browser window at any one time; otherwise changes will be } \\
\text { overwritten. }\end{array}$ \\
\hline Q3: & $\begin{array}{l}\text { Author surnames have been highlighted. Please check these carefully and adjust if the first name or surname is } \\
\text { marked up incorrectly. Note that changes here will affect indexing of your article in public repositories such as } \\
\text { PubMed. Also, carefully check the spelling and numbering of all author names and affiliations, and the corre- } \\
\text { sponding email address(es). }\end{array}$ \\
\hline Q4: & $\begin{array}{l}\text { Please note that after the paper has been formally accepted you can only provide amended Supplementary Infor- } \\
\text { mation files for critical changes to the scientific content, not for style. You should clearly explain what changes } \\
\text { have been made if you do resupply any such files. }\end{array}$ \\
\hline Q5: & Please provide units for time in fig. $2 \mathrm{j}$. \\
\hline Q6: & $\begin{array}{l}\text { The symbol 'pp' is mentioned in Figure } 3 \mathrm{f} \text { legend but is not present in the figure. Please indicate its position in } \\
\text { the figure. }\end{array}$ \\
\hline Q7: & $\begin{array}{l}\text { The symbol 'bm' is mentioned in Figure } 3 \mathrm{f} \text { legend but is not present in the figure. Please indicate its position in } \\
\text { the figure. }\end{array}$ \\
\hline Q8: & Is the insertion of 'Tris-buffered saline' as the definition of 'TBS' correct? \\
\hline Q9: & Is the insertion of 'fetal bovine serum' as the definition of 'FBS' correct? \\
\hline Q10: & 'A.G.' has been changed to 'A.G.-N.' to match the author name on the title page. Please confirm or correct. \\
\hline Q11: & 'J.C.' has been changed to 'J.M.C.' to match author name on title page. Please confirm or correct. \\
\hline Q12: & Reference $[16,15,14,13]$ is a duplicate of $[9,5,3,1]$ and hence the repeated version has been deleted. Please check. \\
\hline Q13: & Please provide the page range or article number for reference $11,26,27,34$ and 36. \\
\hline
\end{tabular}




\section{Reporting Summary}

Nature Research wishes to improve the reproducibility of the work that we publish. This form provides structure for consistency and transparency in reporting. For further information on Nature Research policies, see Authors \& Referees and the Editorial Policy Checklist.

\section{Statistical parameters}

When statistical analyses are reported, confirm that the following items are present in the relevant location (e.g. figure legend, table legend, main text, or Methods section).

n/a Confirmed

$\bigotimes$ The exact sample size $(n)$ for each experimental group/condition, given as a discrete number and unit of measurement

$\searrow$ An indication of whether measurements were taken from distinct samples or whether the same sample was measured repeatedly

$\triangle$ The statistical test(s) used AND whether they are one- or two-sided

$\triangle$ Only common tests should be described solely by name; describe more complex techniques in the Methods section.

\A description of all covariates tested

\A description of any assumptions or corrections, such as tests of normality and adjustment for multiple comparisons

$\square$ A full description of the statistics including central tendency (e.g. means) or other basic estimates (e.g. regression coefficient) AND variation (e.g. standard deviation) or associated estimates of uncertainty (e.g. confidence intervals)

$\varnothing$ For null hypothesis testing, the test statistic (e.g. $F, t, r$ ) with confidence intervals, effect sizes, degrees of freedom and $P$ value noted Give $P$ values as exact values whenever suitable.

Х $\square$ For Bayesian analysis, information on the choice of priors and Markov chain Monte Carlo settings

Х $\square$ For hierarchical and complex designs, identification of the appropriate level for tests and full reporting of outcomes

Х $\square$ Estimates of effect sizes (e.g. Cohen's $d$, Pearson's $r$ ), indicating how they were calculated

$\varnothing$ Clearly defined error bars

State explicitly what error bars represent (e.g. SD, SE, CI)

Our web collection on statistics for biologists may be useful.

\section{Software and code}

Policy information about availability of computer code

Data collection SA3800 version 2.0.4 (SONY) was used for flow citometry. FACSDiva version 8.0.1 (BD Biosciencies) was used for fluorescence-activated cell sorting. Zeiss ZEN 2012 SP5 FP1 version 14.0.11.201 and Leica LAS AF vesion 2.6.3.8173 were used for confocal microscopy. Cell^D version 3.2 and LAS EZ version 3.4.0 were used for optical microscopy. QuantStudio Real time PCR software was used for qPCR data collection.

Data analysis

FlowJo version 10 was used for flow cytometry data analysis. ImageJ version 2006.02.01 was used for image processing. MATLAB version 9.1.0.441655 (R2016b) was used. Matlab analysis procedures that were employed to calculate the young modulus of the CAM and to quantify immunofluorescence images can be made available upon request to the corresponding author. A description of the procedures is available in Methods section of the manuscript. Microsoft excel was used for qPCR and RNA-seq data analysis. Graphpad Prism version 6.01 was used for graphing and statistical analysis. FastQC version 0.11.5, Skewer version 0.2.2, STAR mapper version 2.5.3a and $R$ statistical package DESeq2 were used for RNA-seq data analysis. 
Policy information about availability of data

All manuscripts must include a data availability statement. This statement should provide the following information, where applicable:

- Accession codes, unique identifiers, or web links for publicly available datasets

- A list of figures that have associated raw data

- A description of any restrictions on data availability

RNA-seq data are publicly available in Gene Expression Omnibus (GEO, http://www.ncbi.nlm.nih.gov/geo) under the accession numbers GSE108349, GSE108350 and GSE108351. All remaining datasets that support the findings of this study are available within the article and its supplementary information files, and from the corresponding author upon reasonable request.

\section{Field-specific reporting}

Please select the best fit for your research. If you are not sure, read the appropriate sections before making your selection.

$\bigotimes$ Life sciences $\quad \square$ Behavioural \& social sciences $\quad \square$ Ecological, evolutionary \& environmental sciences

For a reference copy of the document with all sections, see nature.com/authors/policies/Reportingsummary-flat.pdf

\section{Life sciences study design}

All studies must disclose on these points even when the disclosure is negative.

Sample size No statistical methods were used to determine sample size. A minimum of two independent experiements were carried out.

For in vitro experiments, 2-6 organoids were analyzed at the time points and conditions indicated in the manuscript for each experiment. Sample size was determined based on previous studies in the field.

For ex vivo reaggregation assay, we used 1-3 pregnant mice (to collect embryonic kidneys from mice embryos) per experiment. A number of 2-3 reaggregates were analyzed per experiment. Sample size was determined based on previous studies in the field.

For in vivo implantation of organoids, a number of about 6-22 chicken eggs were used per experiment. A number of 2-10 implanted kidney organoids were analyzed per experiment from a total of six experiments. Sample size was determined based on previous studies using tumoral cell lines in the chick CAM. model.

All sample sizes, statistical tests and P values are indicated in the figure legends or described in the "Statistics and reproducibility" section of the manuscript.

Data exclusions No data were excluded from the analyses.

\begin{tabular}{l|l} 
Replication & All experimental findings were reproduced independently at least two times. All attempts at replication were successful. To ensure the
\end{tabular} reproducibility of our methodology for generating kidney organoids, we used three different human embryonic stem cell (hESC) lines and one human induced pluripotent stem cell line.

The number of times that each experiment was repeated is indicated in the figure legends or described in the "Statistics and reproducibility" section of the manuscript.

Randomization Cells/organoids were chosen at random for measurements within each condition.

Blinding Blinding was not used during data collection and analysis in experiments that did not involve direct comparisons between groups. Blinding was used for quantitative measurements comparing different conditions in which data analysis was carried out using a custom made code (Fig. 2g,h; Fig. 2m, n; Fig. 4a,b; Fig.4c,d).

\section{Reporting for specific materials, systems and methods}

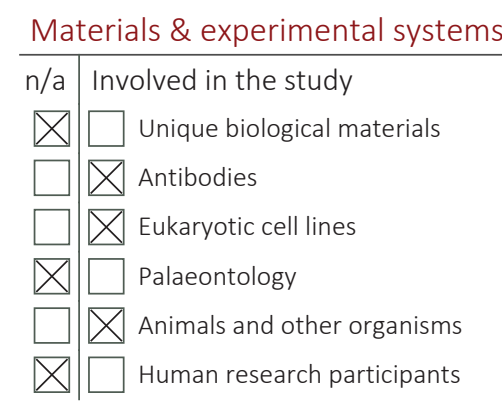

\begin{tabular}{l|l}
\multicolumn{2}{l}{ Methods } \\
\hline n/a Involved in the study \\
$\square$ ChIP-seq \\
$\square$ Mrow cytometry
\end{tabular}


PAX8 (Proteintech, Cat\# 10336-1-AP, polyclonal, Dilution 1:500);

ECAD (BD Bioscience, Cat\# 610181, clone 36/E-cadherine, Lot\# 7187865, Dilution 1:50);

PODXL (R\&D Systems, Cat\# BAF1658, polyclonal, Lot\# JLV0112111, Dilution 1:25);

HNF1ß (Santa Cruz, Cat\# sc-7411, clone C-20, Lot\# B0116, Dilution 1:100);

BRN1 (Santa Cruz, Cat\# sc-6028-R, clone C-17, Lot\# J2512, Dilution 1:200);

NEPHRIN (R\&D Systems, Cat\# AF4269, polyclonal, Lot\# ZMU0114031, Dilution 1:300);

KIM1 (R\&D Systems, Cat\# AF1750, polyclonal, Lot\# JTB0317031, Dilution 1:300);

cleaved Caspase-3 (Cell Signalling, Cat\# 9661S, clone D175, Lot\# 45, Dilution 1:200);

CD34 (Abcam, Cat\# ab8536, cloneQBEND-10, Lot\# GR49632-21, Dilution 1:200);

CD31 (Abcam, Cat\# ab28364, polyclonal, Lot\# GR31176844-16, Dilution 1:50);

HuNu (Abcam, Cat\# ab191181, clone 235-1, Lot\# GR3185051-2, Dilution 1:100);

Uromodulin (UMOD) (R\&D Systems, Cat\# AF5144, polyclonal, Lot\# CBRF0114081, Dilution 1:50);

Aquaporin 1 (AQP1) (Santa Cruz, Cat\# sc-20810, clone H-55, Lot\# C1815, Dilution 1:50);

SLC3A1 (Sigma, Cat\# HPA038360, polyclonal, Lot\# R35388, Dilution 1:100);

Laminin (Sigma, Cat\# L9393, polyclonal, Lot\# 028M4890V, Dilution 1:50);

PODOCIN (Sigma, Cat\# P0372, polyclonal, Lot\# 064M4780, Dilution 1:50);

NEPH1 (Santa Cruz, Cat\# Sc-373787, clone F-6, Lot\# A0313, Dilution 1:50);

SGLT2 (Abcam, Cat\# ab37296, polyclonal, Lot\# GR320725-7, Dilution 1:100);

Sodium potassium ATPase (NaK) (Abcam, Cat\# ab209299, clone ED1845Y, Lot\# GRZ64184-1, Dilution 1:200);

Biotinylated Lotus Tetragonolobus Lectin (LTL) (Vector Laboratories, Cat\# B-1325, Lot\# ZC2428, Dilution 1:200);

Biotinylated Lens Culinaris Agglutinin (LCA) (Vector Laboratories, Cat\# B-1045, Lot\# ZC1221, Dilution 1:500);

Alexa Fluor 488-conjugated streptavidin (Vector Laboratories, Cat\# SA5488, Lot\# ZD0313, Dilution: 1:50);

Antibodies and other staining reagents used for flow cytometry:

Fluorescein labeled LTL (Vector Laboratories, Cat\# FL-1321, Lot\# ZC0914, Dilution: 1:500);

OCT4 conjugated to Alexa Fluor-488 (BD Pharmigen, Cat\# 560253, Lot\# 7110598, Dilution: $20 \mu \mathrm{l} /$ test);

Alexa Fluor 488 Mouse lgG1k isotype control (BD Pharmigen, Cat\# 557721, Lot\# 7082749, Dilution: $5 \mu l /$ test);

Brachyury conjugated to allophycocyanin (APC) (R\&D Systems, Cat\# IC2085A, Lot\# ADUQ0216041, Dilution: $10 \mu l /$ test);

Goat IgG APC-conjugated antibody (R\&D Systems, Cat\# IC108A, Lot\# AAOE0516031, Dilution: $10 \mu \mathrm{l} /$ test);

PAX2 conjugated to A488 using the Lightning-Link ${ }^{\circledR}$ Rapid conjugation kit (322-0010, Innova Biosciences) (R\&D Systems, Cat\# AF3364, Lot\# XOT0215072, Dilution: 1:200);

Normal Goat IgG Alexa Fluor ${ }^{\circledR}$ 488-conjugated Control (R\&D Systems, Cat\# IC108G, Lot\# ABWO41607, Dilution: 5 l/test); LIVE/DEAD Fixable Violet stain reagent (Life Technologies, Cat\# L23105, Dilution: 1:1000)

Information of all antibodies / staining reagents is provided in the Methods section or Supplementary information of the manuscript.

Antibody validations for the species and assay used were performed by antibody suppliers as described in the manufacture's web page, or were published in previous studies. Relevant articles are:

Xia Y, Nivet E, Sancho-Martinez I, Gallegos T, Suzuki K, Okamura D, Wu MZ, Dubova I, Esteban CR, Montserrat N, Campistol JM, Izpisua Belmonte JC. Directed differentiation of human pluripotent cells to ureteric bud kidney progenitor-like cells. Nat Cell Biol. 2013 Dec;15(12):1507-15. doi: 10.1038/ncb2872. Epub 2013 Nov 17. PubMed PMID: 24240476. (OCT4, OSR1, SIX2: human, immunofluorescence).

Martí M, Mulero L, Pardo C, Morera C, Carrió M, Laricchia-Robbio L, Esteban CR, Izpisua Belmonte JC. Characterization of pluripotent stem cells. Nat Protoc. 2013 Feb;8(2):223-53. doi: 10.1038/nprot.2012.154. Epub 2013 Jan 10. PubMed PMID: 23306458. (OCT4, Nanog, Brachyury: human, immunofluorescence).

Elosegui-Artola A, Andreu I, Beedle AEM, Lezamiz A, Uroz M, Kosmalska AJ, Oria R, Kechagia JZ, Rico-Lastres P, Le Roux AL, Shanahan CM, Trepat X, Navajas D, Garcia-Manyes S, Roca-Cusachs P. Force Triggers YAP Nuclear Entry by Regulating Transport across Nuclear Pores. Cell. 2017 Nov 30;171(6):1397-1410.e14. doi: 10.1016/j.cell.2017.10.008. Epub 2017 Oct 26. PubMed PMID: 29107331. (YAP: human, immunofluorescence)

Morizane R, Lam AQ, Freedman BS, Kishi S, Valerius MT, Bonventre JV. Nephron organoids derived from human pluripotent stem cells model kidney development and injury. Nat Biotechnol. 2015 Nov;33(11):1193-200. PubMed PMID: 26458176; PubMed Central PMCID: PMC4747858. (SALL1, LHX1, SIX2, PAX8, PODXL, HNF1ß, BRN1, KIM1, LTL: human, immunofluorescence)

Takasato M, Er PX, Chiu HS, Maier B, Baillie GJ, Ferguson C, Parton RG, Wolvetang EJ, Roost MS, Chuva de Sousa Lopes SM, Little MH. Kidney organoids from human iPS cells contain multiple lineages and model human nephrogenesis. Nature. 2015 Oct 
Sharmin S, Taguchi A, Kaku Y, Yoshimura Y, Ohmori T, Sakuma T, Mukoyama M, Yamamoto T, Kurihara H, Nishinakamura R. Human Induced Pluripotent Stem Cell-Derived Podocytes Mature into Vascularized Glomeruli upon Experimental Transplantation. J Am Soc Nephrol. 2016 Jun;27(6):1778-91. doi: 10.1681/ASN.2015010096. Epub 2015 Nov 19. PubMed PMID: 26586691; PubMed Central PMCID: PMC4884101. (CD31: human, immunofluorescence).

Cai G, Lai B, Hong H, Lin P, Chen W, Zhu Z, Chen H. Effects of cryopreservation on excretory function, cellular adhesion molecules and vessel lumen formation in human umbilical vein endothelial cells. Mol Med Rep. 2017 Jul;16(1):547-552. doi: 10.3892/ mmr.2017.6664. Epub 2017 May 31. PubMed PMID:28586042; PubMed Central PMCID: PMC5482135. (CD34: human, immunofluorescence).

Lei P, Ding D, Xie J, Wang L, Liao Q, Hu Y. Expression profile of Twist, vascular endothelial growth factor and CD34 in patients with different phases of osteosarcoma. Oncol Lett. 2015 Jul;10(1):417-421. Epub 2015 May 20. PubMed PMID: 26171042; PubMed Central PMCID: PMC4487166. (CD34: human, immunofluorescence).

Cruz NM, Song X, Czerniecki SM, Gulieva RE, Churchill AJ, Kim YK, Winston K, Tran LM, Diaz MA, Fu H, Finn LS, Pei Y, Himmelfarb J, Freedman BS. Organoid cystogenesis reveals a critical role of microenvironment in human polycystic kidney disease. Nat Mater. 2017 Nov;16(11):1112-1119. doi: 10.1038/nmat4994. Epub 2017 Oct 2. PubMed PMID: 28967916; PubMed Central PMCID: PMC5936694. (NEPHRIN, PODXL, LTL, Fluorescein labeled LTL: human, immunofluorescence).

Lindström NO, Tran T, Guo J, Rutledge E, Parvez RK, Thornton ME, Grubbs B, McMahon JA, McMahon AP. Conserved and Divergent Molecular and Anatomic Features of Human and Mouse Nephron Patterning. J Am Soc Nephrol. 2018 Mar;29(3):825-840. doi: 10.1681/ASN.2017091036. Epub 2018 Feb 15. PubMed PMID: 29449451; PubMed Central PMCID: PMC5827611. (Pax2, WT1, SLC3A1, Uromodulin: human, immunofluorescence).

Vedula EM, Alonso JL, Arnaout MA, Charest JL. A microfluidic renal proximal tubule with active reabsorptive function. PLoS One. 2017 Oct 11;12(10): e0184330. doi: 10.1371/journal.pone.0184330. eCollection 2017. PubMed PMID: 29020011; PubMed Central PMCID: PMC5636065. (SGLT2: human, immunofluorescence).

Kang KJ, Lee MS, Moon CW, Lee JH, Yang HS, Jang YJ. In Vitro and In Vivo Dentinogenic Efficacy of Human Dental Pulp-Derived Cells Induced by Demineralized Dentin Matrix and HA-TCP. Stem Cells Int. 2017; 2017:2416254. doi:10.1155/2017/2416254. Epub 2017 Jun 28. PubMed PMID: 28761445; PubMed Central PMCID: PMC5518496. (HuNu: human, immunofluorescence).

Jilani SM, Murphy TJ, Thai SN, Eichmann A, Alva JA, Iruela-Arispe ML. Selective binding of lectins to embryonic chicken vasculature. J Histochem Cytochem. 2003 May;51(5):597-604. PubMed PMID: 12704207. (Biotinylated Lens Culinaris Agglutinin: chicken, immunofluorescence).

Yamaguchi S, Morizane R, Homma K, Monkawa T, Suzuki S, Fujii S, Koda M, Hiratsuka K, Yamashita M, Yoshida T, Wakino S, Hayashi K, Sasaki J, Hori S, Itoh H. Generation of kidney tubular organoids from human pluripotent stem cells. Sci Rep. 2016 Dec 16;6: 38353. doi: 10.1038/srep38353.PubMed PMID: 27982115; PubMed Central PMCID: PMC5159864. (Aquaporin 1: human, immunofluorescence).

Fagerberg L, Hallström BM, Oksvold P, Kampf C, Djureinovic D, Odeberg J, Habuka M, Tahmasebpoor S, Danielsson A, Edlund K, Asplund A, Sjöstedt E, Lundberg E, Szigyarto CA, Skogs M, Takanen JO, Berling H, Tegel H, Mulder J, Nilsson P, Schwenk JM, Lindskog C, Danielsson F, Mardinoglu A, Sivertsson A, von Feilitzen K, Forsberg M, Zwahlen M, Olsson I, Navani S, Huss M, Nielsen J, Ponten F, Uhlén M. Analysis of the human tissue-specific expression by genome-wide integration of transcriptomics and antibody-based proteomics. Mol Cell Proteomics. 2014 Feb;13(2):397-406. doi: 10.1074/mcp.M113.035600. Epub 2013 Dec 5. PubMed PMID: 24309898; PubMed Central PMCID: PMC3916642. (PODOCIN: human, immunofluorescence).

Wong KG, Ryan SD, Ramnarine K, Rosen SA, Mann SE, Kulick A, De Stanchina E, Müller FJ, Kacmarczyk TJ, Zhang C, Betel D, Tomishima MJ. CryoPause: A New Method to Immediately Initiate Experiments after Cryopreservation of Pluripotent Stem Cells. Stem Cell Reports. 2017 Jul 11;9(1):355-365. doi: 10.1016/j.stemcr.2017.05.010. Epub 2017 Jun 8. PubMed PMID: 28602613; PubMed Central PMCID: PMC5511100. (Brachyury conjugated to allophycocyanin (APC): human, flow cytometry).

\section{Eukaryotic cell lines}

Policy information about cell lines

Cell line source(s)
Human pluripotent stem cell lines:

All the human pluripotent stem cell lines used in this study were obtained after the approval of the Ethics Committee of the CMRB and the approval of the Comisión de Seguimiento y Control de la Donación de Células y Tejidos Humanos del Instituto de Salud Carlos III (project numbers: 0336E/7564/2016; 0336E/5311/2015; 0336E/15986/2016; 0336E/79489/2015; 00336E/20031/2014). ES[4] hESC and CBiPSsv-4F-40 were obtained from The National Bank of Stem Cells (ISCIII, Madrid). H1 and $\mathrm{H} 9 \mathrm{hESC}$ lines were purchased at Wicell.

Information on cell line sources used in this study is also provided in the Methods section of the manuscript.

Human kidney material:

Primary human proximal tubular cells were obtained from collaborators at Hospital Clinic de Barcelona, Spain. The ethics committee of Hospital Clinic de Barcelona approved the procedure, and signed consent forms are available upon request (project number: 2009/5023). Isolation of primary renal proximal tubular epithelial cells was performed as previously described. Briefly, tubular cells were prepared from renal tissue after nephrectomy. Cortical renal tissue was digested in 
Iscove's modified Dulbecco's medium with 1\% collagenase IV (Invitrogen) for $1 \mathrm{~h}$ under shaking. Cell suspension was placed on a pre-cooled Percoll density gradient solution (Amersham Biosciences) and centrifuged for $40 \mathrm{~min}$ at $4{ }^{\circ} \mathrm{C}$ at $16,000 \mathrm{rpm}$ (gradient with densities between 1.019 and $1.139 \mathrm{~g} / \mathrm{ml}$ was generated). The cell fraction between 1.05 and $1.076 \mathrm{~g} / \mathrm{ml}$ was collected and cells were plated on plastic plates with DMEM (Invitrogen) supplemented with 10\% fetal bovine serum (FBS; Invitrogen), Glutamax (1 mM), penicillin/streptomycin, and nonessential amino acids (100 $\mu \mathrm{M})$.

Relevant reference:

Montserrat N, Ramírez-Bajo MJ, Xia Y, Sancho-Martinez I, Moya-Rull D, Miquel-Serra L, Yang S, Nivet E, Cortina C, González F, Izpisua Belmonte JC, Campistol JM. Generation of induced pluripotent stem cells from human renal proximal tubular cells with only two transcription factors, OCT4 and SOX2. J Biol Chem. 2012 Jul 13;287(29):24131-8.

Human fetal kidney samples included in this study were provided by the Fetal Tissue Bank of Vall d'Hebron University Hospital Biobank (PT13/0010/0021), integrated in the Spanish National Biobanks Network and they were processed following standard operating procedures with the appropriate approval of the Ethical and Scientific Committees (project number: 0336E/9934/2015). Human fetal kidney samples from 13, 16 and 22 weeks of gestation were supplied as whole tissues embedded in OCT (at -80 ㅇ) and as frozen tissue samples for RNA extraction.

Information on human kidney material used in this study is also provided in the Methods section of the manuscript.

Authentication

Mycoplasma contamination

Commonly misidentified lines (See ICLAC register)
Human pluripotent stem cell lines:

Human embryonic stem cells (ES4, $\mathrm{H} 1$ and $\mathrm{H} 9$ lines) and human induced pluripotent stem cells (CBiPSsv-4F-40 line) were authenticated in their lab of origin through the expression of pluripotency-associated markers.

Human kidney material:

Primary human proximal epithelial cells were authenticated in the lab of origin for the expression of specific proximal tubular cell markers, as previously reported.

Relevant reference:

Montserrat N, Ramírez-Bajo MJ, Xia Y, Sancho-Martinez I, Moya-Rull D, Miquel-Serra L, Yang S, Nivet E, Cortina C, González F, Izpisua Belmonte JC, Campistol JM. Generation of induced pluripotent stem cells from human renal proximal tubular cells with only two transcription factors, OCT4 and SOX2. J Biol Chem. 2012 Jul 13;287(29):24131-8.

The gestational age of human fetal kidney samples was determined per guidelines specified by the American College of Obstetricians and Gynecologists using ultrasound heel-to-toe, and crown-to-rump measurements.

Relevant reference:

O'Rahilly R, Müller F: Developmental stages in human embryos: Revised and new measurements. Cells Tissues Organs 192: 73-84, 2010

All cell lines tested negative for mycoplasma contamination.

No commonly misidentified cell lines were used.

\section{Animals and other organisms}

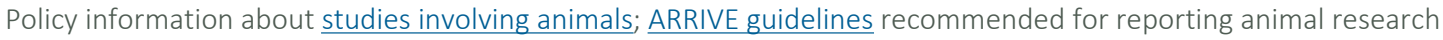

Laboratory animals

Wild animals

Field-collected samples
For mice experiments:

Animal care and experiments were carried out according to protocols approved by the Ethics Committee on Animal Research of the University of Barcelona, Spain (protocol number: OB 391/18). Embryonic kidneys from 11.5-12.5 days post conception (d.p.c) were collected from time-mated pregnant C57BL/6J mice.

For chicken experiments:

According to animal care guidelines in Spain, no approval was necessary to perform the experiments described herein. Fertilized white Leghorn chicken eggs were provided by Granja Gibert, rambla Regueral, S/N, 43850 Cambrils, Spain.

The study does not involve wild animals.

The study does not involve samples collected from the field.

\section{Flow Cytometry}

\section{Plots}

Confirm that:

Х The axis labels state the marker and fluorochrome used (e.g. CD4-FITC).

Х The axis scales are clearly visible. Include numbers along axes only for bottom left plot of group (a 'group' is an analysis of identical markers).

\ All plots are contour plots with outliers or pseudocolor plots.

Х A numerical value for number of cells or percentage (with statistics) is provided.

\section{Methodology}


Sample preparation

Instrument

Software

Cell population abundance

Gating strategy methodology described in the Methods section of the manuscript.

Kidney organoids were stained according to a standard methodology described in the Methods section of the manuscript. Kidney organoids were then dissociated to single cells using accumax (07921, Stem Cell Technologies) for 15 min followed by $0.25 \%$ (wt/ vol) trypsin (25300-054, Life Technologies) for 15 min at 37으.

Sony SA3800 spectral cell analyzer (SONY) was used to acquire flow cytometry samples (Supplementary Fig.1b and Supplementary Fig.2b), whereas FACSAria Fusion instrument (BD Biosciences) was used for cell sorting experiments (Supplementary Fig. 5b).

SA3800 software version 2.0.4 (SONY) was used to acquire flow cytometry samples in the Sony SA3800 spectral cell analyzer (SONY), whereas FACSDiva software version 8.0.1 (BD Biosciencies) was used in the FACS Aria Fusion instrument (BD Biosciences) for cell sorting experiments. FlowJo software version 10 was used to analyze these data.

The abundance of the relevant cell populations was based on the expression of specific nuclear and surface markers that were analyzed by immunofluorescence and quantitative RT-PCR.

Supplementary Fig. 1b:

For assessment of OCT4 and T staining, cells were first gated on a SS-A versus FS-A plot, the polulation from which was then gated on the LIVE/DEAD negative population (LIVE/DEAD versus FS-A plot), the population from which was then analyzed on a plot of T-APC versus OCT4-A488.

Supplementary Fig. 2b:

For assessment of PAX2 staining, cells were first gated on a SSC-A versus FSC-A plot, the polulation from which was then gated on the LIVE/DEAD negative population (LIVE/DEAD versus FSC-A plot), the population from which was then analyzed on a plot of SSC-A versus PAX2-A488.

Supplementary Fig. 5b:

For fluorescence activated cell sorting of LTL-FITC positive and negative cell fractions, cells were first gated on a SSC-A versus FSC-A plot, the polulation from which was then gated on singlets (FSC-W versus FSC-A plot), the population from which was then gated on the LIVE/DEAD negative population (LIVE/DEAD versus FSC-A plot), the population from which was then analyzed on a plot of autofluorescence (AU) versus LTL-FITC.

$\bigotimes$ Tick this box to confirm that a figure exemplifying the gating strategy is provided in the Supplementary Information. 